\title{
Dynamical twisted mass fermions with light quarks: simulation and analysis details
}

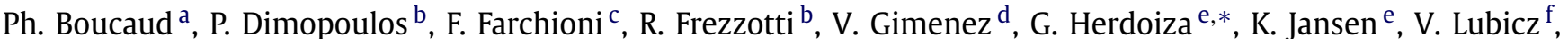

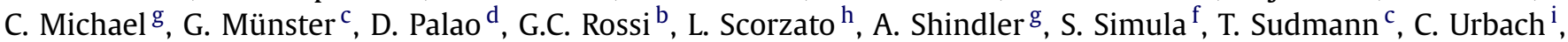 \\ U. Wenger ${ }^{\mathrm{j}}$
}

\footnotetext{
a Laboratoire de Physique Théorique (Bât. 210), Université de Paris XI, Centre d'Orsay, 91405 Orsay-Cedex, France

b Dip. di Fisica, Università di Roma Tor Vergata and INFN Sez. di Roma Tor Vergata, Via della Ricerca Scientifica 1, I-00133 Roma, Italy

c Universität Münster, Institut für Theoretische Physik, Wilhelm-Klemm-Strasse 9, D-48149 Münster, Germany

d Dep. de Física Teòrica and IFIC, Universitat de València-CSIC, Dr. Moliner 50, E-46100 Burjassot, Spain

e DESY Zeuthen, Platanenallee 6, D-15738 Zeuthen, Germany

${ }^{f}$ Dip. di Fisica, Università di Roma Tre and INFN, Sez. di Roma III, Via della Vasca Navale 84, I-00146 Roma, Italy

g Theoretical Physics Division, Department of Mathematical Sciences, University of Liverpool, Liverpool L69 7ZL, UK

${ }^{\mathrm{h}}$ ECT $^{*}$ and INFN, strada delle tabarelle, 286 - 38100 Trento, Italy

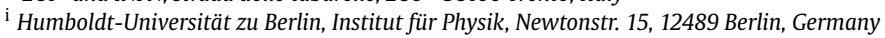

j Institute for Theoretical Physics, ETH Zürich, $\mathrm{CH}-8093$ Zürich, Switzerland
}

\section{A R T I C L E I N F O}

\section{Article history:}

Received 3 March 2008

Received in revised form 26 May 2008

Accepted 15 June 2008

Available online 3 July 2008

\section{PACS:}

12.38.GC

12.39.Fe

14.40.Aq

14.65.Bt

\section{Keywords:}

Lattice gauge theory

Lattice QCD

Hybrid Monte Carlo algorithm

Stochastic quark propagators

\begin{abstract}
A B S T R A C T
In a recent paper [ETMC, P. Boucaud et al., Phys. Lett. B 650 (2007) 304, hep-lat/0701012] we presented precise lattice QCD results of our European Twisted Mass Collaboration (ETMC). They were obtained by employing two mass-degenerate flavours of twisted mass fermions at maximal twist. In the present paper we give details on our simulations and the computation of physical observables. In particular, we discuss the problem of tuning to maximal twist, the techniques we have used to compute correlators and error estimates. In addition, we provide more information on the algorithm used, the autocorrelation times and scale determination, the evaluation of disconnected contributions and the description of our data by means of chiral perturbation theory formulae.
\end{abstract}

(c) 2008 Elsevier B.V. All rights reserved.

\section{Twisted mass fermions}

Dynamical Wilson twisted mass fermions, when tuned to maximal twist [2,3], have been demonstrated to lead to precise results for mesonic quantities down to pseudo scalar masses $m_{\mathrm{PS}} \lesssim 300 \mathrm{MeV}$. Results in the quenched case were discussed in Refs. [4-6] and in the case of two mass-degenerate flavours of quarks in Ref. [1]. Preparatory simulations with twisted mass dynamical fermions were performed in [7-10]. In Ref. [1] many of the details of our computations had to be omitted and it is the purpose of the present paper to supplement those and fill this gap.

\footnotetext{
* Corresponding author.

E-mail address: gregorio.herdoiza@desy.de (G. Herdoiza).
}

This paper is organised as follows. In this section we introduce twisted mass fermions and discuss the important issue of tuning to maximal twist. In Section 2, we give details about our techniques to compute charged correlators and in Section 3 to compute neutral correlators and quark-disconnected contributions. In Section 4 we discuss the algorithm details and explain our analysis techniques for obtaining reliable error estimates. In Section 5 we provide details of our computation of the force parameter $r_{0}$ and in Section 6 we give some results for the pseudoscalar mass and decay constant, the untwisted PCAC quark mass and the renormalisation constant $Z_{\mathrm{V}}$. We use chiral perturbation theory to fit our data and we detail this procedure in Section 7. We end with a short summary in Section 8.

We begin with the Wilson twisted mass fermionic lattice action for two flavours of mass degenerate quarks, which reads (in 
the so-called twisted basis [2,3] and with fermion fields having continuum dimensions)

$S_{\mathrm{tm}}^{\mathrm{F}}=a^{4} \sum_{x}\left\{\bar{\chi}_{x}\left[D_{\mathrm{W}}+m_{0}+i \gamma_{5} \tau_{3} \mu_{q}\right] \chi_{x}\right\}$,

$D_{\mathrm{W}}=\frac{1}{2} \gamma_{\mu}\left(\nabla_{\mu}+\nabla_{\mu}^{*}\right)-\frac{a r}{2} \nabla_{\mu} \nabla_{\mu}^{*}$,

where $m_{0}$ is the bare untwisted quark mass and $\mu_{q}$ the bare twisted quark mass, $\tau_{3}$ is the third Pauli matrix acting in flavour space and $r$ is the Wilson parameter, which we set to $r=1$ in our simulations. We denote by $\nabla_{\mu}$ and $\nabla_{\mu}^{*}$ the gauge covariant nearest neighbour forward and backward lattice derivatives. The bare quark mass $m_{0}$ is related to the so-called hopping parameter $\kappa$, which we will often use in this paper, by $\kappa=1 /\left(8+2 a m_{0}\right)$. Twisted mass fermions are said to be at maximal twist if the bare untwisted mass is tuned to its critical value, $m_{\text {crit }}$. We will discuss later how this can be achieved in practice.

In the gauge sector we use, for reasons explained in [1], the so-called tree-level Symanzik improved gauge action (tlSym) [11] which includes besides the plaquette term $U_{x, \mu, v}^{1 \times 1}$ also rectangular $(1 \times 2)$ Wilson loops $U_{x, \mu, v}^{1 \times 2}$. It reads

$$
\begin{gathered}
S_{g}=\frac{\beta}{3} \sum_{x}\left(b_{0} \sum_{\substack{\mu, \nu=1 \\
1 \leqslant \mu<v}}^{4}\left\{1-\operatorname{Re} \operatorname{Tr}\left(U_{x, \mu, \nu}^{1 \times 1}\right)\right\}\right. \\
\left.+b_{1} \sum_{\substack{\mu, \nu=1 \\
\mu \neq \nu}}^{4}\left\{1-\operatorname{Re} \operatorname{Tr}\left(U_{x, \mu, \nu}^{1 \times 2}\right)\right\}\right),
\end{gathered}
$$

where $\beta$ is the bare inverse coupling and we set $b_{1}=-1 / 12$ (with $b_{0}=1-8 b_{1}$ as dictated by the requirement of continuum limit normalisation). Note that at $b_{1}=0$ this action becomes the usual Wilson plaquette gauge action.

\subsection{Tuning to maximal twist}

One of the main virtues of Wilson twisted mass fermions is that by tuning the bare quark mass $m_{0}$ to its critical value an automatic $\mathrm{O}(a)$ improvement can be achieved such that expectation values of parity even operators scale to their continuum limit with $\mathrm{O}\left(a^{2}\right)$ discretisation errors [3]. It was shown in the scaling test study carried out in [4-6] in the quenched case that $\mathrm{O}(a)$ improvement works extremely well for maximally twisted mass quarks. In this context, the method to tune to maximal twist by setting the so-called (untwisted) PCAC mass to zero (in the limit $\mu_{q} \rightarrow 0$ ) was found to be very successful, in agreement with theoretical considerations [12-14]. In the present paper essentially the same approach to set to zero the (untwisted) PCAC mass

$m_{\mathrm{PCAC}}=\frac{\sum_{\mathbf{x}}\left\langle\partial_{0} A_{0}^{a}(\mathbf{x}, t) P^{a}(0)\right\rangle}{2 \sum_{\mathbf{x}}\left\langle P^{a}(\mathbf{x}, t) P^{a}(0)\right\rangle}, \quad a=1,2$,

was followed, by evaluating (3) at large enough time separation, so that the pion ground state is dominant. For a definition of the (twisted basis) operators appearing in Eq. (3) see Eq. (A.1) of Appendix A.

In principle one could think of determining $a m_{\text {crit }}$ at each value of $a \mu_{q}$ at which simulations are carried out and then perform an extrapolation to vanishing $a \mu_{q}$ based on data satisfying the bound $a \mu_{q} \gtrsim a^{3} \Lambda_{\mathrm{QCD}}^{3}$ [14]. This method is, however, rather CPU-time expensive. We therefore prefer to determine the value of $a m_{\text {crit }}$ (at each fixed value of $\beta$ ) from the simulation at the lowest available value, $a \mu_{q, \min } \ll a \Lambda_{\mathrm{QCD}}$. This choice simply affects the critical quark mass by $\mathrm{O}\left(a \mu_{q, \min } \Lambda_{\mathrm{QCD}}\right)$ terms. Therefore $\mathrm{O}(a)$ improvement is still guaranteed [3]. Furthermore, and most importantly, with such a determination of $a m_{\text {crit }}$ also the $\mathrm{O}\left(a^{2}\right)$ cutoff effects remain small as long as $\mu_{q} \gtrsim a^{2} \Lambda_{\mathrm{QCD}}^{3}$ [14]. We recall below the line of arguments leading to this conclusion.

\subsection{Maximal twist and residual $\mathrm{O}\left(a^{2}\right)$ artifacts}

To start the discussion let us assume that $m_{0}=1 /(2 \kappa)-4$ has been set to a value corresponding to some sensible lattice estimate of the critical mass, while $\mu_{q}$ is non-zero. In this situation one is already at maximal twist. However the unavoidable $\mathrm{O}(a)$ terms affecting any determination of the critical mass can be further tuned in an "optimal way", i.e. in a way such that the residual $\mathrm{O}\left(a^{2}\right)$ lattice artifacts in physical quantities remain under control as the pion mass is decreased. We briefly explain how this can be achieved in practice and to what accuracy, following the work of Ref. [14]. In the Symanzik expansion of the lattice expectation value $\left.\langle O(x)\rangle\right|_{\mu_{q}}$ of a multilocal operator $O$ computed at a bare quark mass $\mu_{q}$ there will appear at $\mathrm{O}\left(a^{2}\right)$ terms which are proportional to

$\left[\frac{1}{m_{\pi}^{2}}\right]^{2} \xi_{\pi}^{2}\left(\mu_{q}\right) \propto \frac{1}{\mu_{q}^{2}} \xi_{\pi}^{2}\left(\mu_{q}\right)$,

where

$\xi_{\pi}\left(\mu_{q}\right)=\left|\left\langle\Omega\left|\mathcal{L}_{\text {odd }}\right| \pi^{0}(\mathbf{0})\right\rangle\right|_{\mu_{q}}^{\text {cont }}$.

Here $\langle\Omega|$ and $\left|\pi^{0}(\mathbf{0})\right\rangle$ denote the vacuum and the one-pion neutral state at zero three-momentum, respectively. With the symbol

$\mathcal{L}_{\text {odd }}=a \mathcal{L}_{5}+a^{3} \mathcal{L}_{7}+\cdots$

we indicate the set of operators of odd dimension in the Symanzik local effective Lagrangian that describes the maximally twisted lattice theory. From Eq. (4) one recognises that cut-off effects may become large when $m_{\pi}^{2}$ gets small.

The general strategy to avoid these large cut-off effects is to tune $\xi_{\pi}\left(\mu_{q}\right)$ to zero, or at least to reduce it to $\mathrm{O}\left(\mathrm{am}_{\pi}^{2} \Lambda_{\mathrm{QCD}}^{2}\right)$ by adjusting the value of $\kappa_{\text {crit }}$. One way to realise this is precisely to tune $m_{\text {PCAC }}=0$ as explained above. In particular it is sufficient to impose the vanishing of the PCAC mass at $\mu_{q}=\mu_{q, \text { min }}$ [14]. An analysis à la Symanzik of the correlator in the numerator of Eq. (3) shows that, if $\kappa$ is such that $m_{\mathrm{PCAC}}$ vanishes at a given value of $\mu_{q}$ (provided $\left.\mu_{q}<\Lambda_{\mathrm{QCD}}\right)$, then $\xi_{\pi}\left(\mu_{q}\right)$ is suppressed in a sufficiently strong way, namely one gets (note that $\xi_{\pi}$ has mass dimension three)

$\xi_{\pi}\left(\mu_{q}\right)=\mathrm{O}\left(a \mu_{q} \Lambda_{\mathrm{QCD}}^{3}\right)+\mathrm{O}\left(a \mu_{q}^{2} \Lambda_{\mathrm{QCD}}^{2}\right)+\mathrm{O}\left(a^{3} \Lambda_{\mathrm{QCD}}^{6}\right)$,

and thus (see Eq. (4))

$\frac{\xi_{\pi}\left(\mu_{q}\right)}{\mu_{q} \Lambda_{\mathrm{QCD}}^{2}}=\mathrm{O}\left(a \Lambda_{\mathrm{QCD}}\right)+\mathrm{O}\left(a \mu_{q}\right)+\mathrm{O}\left(a \Lambda_{\mathrm{QCD}} \frac{a^{2} \Lambda_{\mathrm{QCD}}^{3}}{\mu_{q}}\right)$.

In this situation, the ratio $\xi_{\pi}\left(\mu_{q}\right) / \mu_{q} \Lambda_{\mathrm{QCD}}^{2}$ remains small as long as $\mu_{q} \gtrsim a^{2} \Lambda_{\mathrm{QCD}}^{3}$.

For each value of $\mu_{q}$ in the region $a^{2} \Lambda_{\mathrm{QCD}}^{3} \lesssim \mu_{q}<\Lambda_{\mathrm{QCD}} \ll a^{-1}$ the value of $\kappa$ at which $m_{\mathrm{PCAC}}$ vanishes provides a legitimate estimate of $\kappa_{\text {crit }}$ and hence of $m_{\text {crit }}$. Estimates of $m_{\text {crit }}$ corresponding to different values of $\mu_{q}$ differ by $\mathrm{O}\left(a \mu_{q} \Lambda_{\mathrm{QCD}}\right)$ from each other. In particular, working at $\kappa_{\text {crit }}\left(\mu_{q, \text { min }}\right)$ leads to $\mathrm{O}\left(a^{2}\right)$ cutoff effects which are at worst of the form $a^{2}\left(\mu_{q, \min } / \mu_{q}\right)^{2}$ and thus perfectly tolerable as long as $\mu_{q} \gtrsim \mu_{q, \min }>a^{2} \Lambda_{\mathrm{QCD}}^{3}$. This result can be checked by expanding $\xi_{\pi}\left(\mu_{q}\right)$ around $\mu_{q \text {,min }}$ in Eq. (8) and using the expression of $\xi_{\pi}\left(\mu_{q, \text { min }}\right)$ from Eq. (7). 


\subsection{Numerical precision for tuning to maximal twist}

It remains to be discussed to what numerical precision the condition $m_{\text {PCAC }}=0$ has to be fulfilled. This question is important if one wants to avoid that numerical uncertainties jeopardise the tuning procedure. Suppose $\left|a m_{\mathrm{PCAC}}\right|=a \epsilon \neq 0$, where $a \epsilon$ denotes a small deviation, due to numerical limitations, from the condition of vanishing PCAC mass. As a rule of thumb the value of $a \epsilon$ can be taken as the maximum (in modulus) between the finite statistics central value of $a m_{\text {PCAC }}$ and its (estimated) standard deviation. It then follows by expanding $\xi_{\pi}=\xi_{\pi}\left(\mu_{q}, \epsilon\right)$ around $\epsilon=0$

$$
\begin{aligned}
\xi_{\pi}\left(\mu_{q}, \epsilon\right) & =\xi_{\pi}\left(\mu_{q}\right)+\mathrm{O}\left(\Lambda_{\mathrm{QCD}}^{2} \epsilon\right) \\
& \approx \mathrm{O}\left(a \mu_{q} \Lambda_{\mathrm{QCD}}^{3}\right)+\mathrm{O}\left(a \mu_{q}^{2} \Lambda_{\mathrm{QCD}}^{2}\right)+\mathrm{O}\left(\Lambda_{\mathrm{QCD}}^{2} \epsilon\right) .
\end{aligned}
$$

Thus for the relative size of $\xi_{\pi}$ compared to the actual value of the quark mass, one gets

$$
\frac{\xi_{\pi}\left(\mu_{q}, \epsilon\right)}{\mu_{q} \Lambda_{\mathrm{QCD}}^{2}}=\mathrm{O}\left(a \Lambda_{\mathrm{QCD}}\right)+\mathrm{O}\left(a \mu_{q}\right)+\mathrm{O}\left(\frac{\epsilon}{\mu_{q}}\right) .
$$

A smooth approach to the continuum is, of course, guaranteed when $\left|\epsilon / \mu_{q}\right|$ is of order $a \Lambda_{\mathrm{QCD}}$ or smaller. In fact, from the form of the dimension five term in the Symanzik effective Lagrangian of the twisted mass lattice QCD, it follows that, close to maximal twist, $a \Lambda_{\mathrm{QCD}}\left|\epsilon / \mu_{q}\right|$ is the expected order of magnitude of the (unwanted) relative $\mathrm{O}(a)$ cutoff effects stemming from violations of the condition of vanishing PCAC mass. The requirement $\left|\epsilon / \mu_{q}\right| \lesssim$ $a \Lambda_{\mathrm{QCD}}$ thus implies that the relative magnitude of these unwanted cutoff effects satisfies the constraint $a \Lambda_{\mathrm{QCD}}\left|\epsilon / \mu_{q}\right| \lesssim a^{2} \Lambda_{\mathrm{QCD}}^{2}$, which is consistent with $\mathrm{O}(a)$ improvement.

In practice, since we are interested in simulations performed at lattice spacings about (or slightly below) $0.1 \mathrm{fm}$, where $a \Lambda_{\mathrm{QCD}} \sim$ 0.1 , a value of $\left|\epsilon / \mu_{q}\right| \lesssim 0.1$ (and ideally decreasing with $a$ ) will represent an acceptable level of precision in the procedure of determining the critical mass. This condition is not too restrictive as in actual simulations it is sufficient that it holds at $\mu_{q, \min }$. We also remark that in order to check scaling and perform a reliable continuum extrapolation, the value of $\mu_{q \text {,min }}$ should be kept roughly fixed in physical units as the lattice spacing is decreased.

Although these theoretical arguments show that we can work in conditions such that we are effectively left with only $\mathrm{O}\left(a^{2}\right)$ lattice artefacts, numerical computations are required to check the scaling behaviour and determine the order of magnitude of the coefficient multiplying $a^{2}$ terms for the observables of interest. In this paper, where data at only one value of $a$ are analysed, we cannot evaluate these coefficients. Nevertheless, for the observables we discuss here preliminary results from our collaboration presented in Refs. $[15,16]$ indicate that the residual cutoff effects are indeed small and consistent with $\mathrm{O}(a)$ improvement.

\section{Computations in the charged meson sector}

In this paper we will be mainly using the twisted quark basis where the fermionic action takes the form (1). Even though there is no fundamental reason for this choice, employing the twisted quark basis makes immediately transparent the way several computational methods, which have been invented for, or widely applied to, untwisted Wilson fermions, carry over to the case of maximally twisted Wilson quarks. Of course, in such an unphysical basis, the two flavour components of the fermion field $\chi=(u, d)^{T}$ appearing in the action do not coincide with the canonical quark fields in the "physical" basis, $\psi=\left(u_{\text {phys }}, d_{\text {phys }}\right)^{T}$, rather the former are related to the latter by the axial rotation

$$
\begin{aligned}
& \chi=\left.e^{-i \gamma_{5} \tau_{3} \omega / 2}\right|_{\omega=\pi / 2} \psi \quad \Leftrightarrow \quad u=e^{-i \gamma_{5} \pi / 4} u_{\text {phys }}, \\
& d=e^{i \gamma_{5} \pi / 4} d_{\text {phys }},
\end{aligned}
$$

which we write here in the case of maximal twist, $\omega=\pi / 2$. Since the axial transformation above is flavour diagonal, the names of the components $(u, d)$ of the twisted basis field $\chi$ are still appropriate to their flavour content. In spite of that, the correct interpretation of gauge invariant composite bare operators in the $(\chi, \bar{\chi})$ basis is obtained only once they are expressed in terms of the physical basis bare fields $(\psi, \bar{\psi})$. Examples concerning quark bilinear fields can be found in Appendix A.

In this context it may be useful to remark that, since parity and isospin are no longer exact symmetries (recall however that $I_{3}$, the third isospin component, is unbroken), a physical basis bare composite operator with given formal parity and isospin properties can interpolate a hadron with opposite parity and/or different isospin. As a consequence in the quantum-mechanical representation of the correlators there will be contributions containing matrix elements of a physical basis composite operator with given formal parity and isospin between the vacuum and a state with opposite parity and/or different isospin, as well as between a neutral pion state (which has the same lattice quantum numbers as the vacuum) and a state with the same parity and isospin properties as the considered operator. Such parity- and/or isospin-violating matrix elements are of course of order $a$. Their occurrence in the quantum-mechanical representation of correlators is not in contradiction with the $\mathrm{O}(a)$ improvement of the expectation values of parity-even, or isospin-invariant, multilocal operators [3]. For these specific correlators, indeed, an analysis à la Symanzik shows that each term of their quantum-mechanical representation can contain only an even number of $\mathrm{O}(a)$ factors given by parity- and/or isospin-violating matrix elements. ${ }^{1}$

From the formulae in Appendix A, it is clear that at maximal twist, $\omega=\pi / 2$, the operator $\bar{d} \gamma_{5} u$ is associated to the $\pi^{+}$meson, in the sense that $\left(\bar{d} \gamma_{5} u\right)^{\dagger}$ creates the $\pi^{+}$state from the vacuum. The two-point $\pi^{+}$meson correlator receives contributions only from (fermionically) connected diagrams, and after integration over fermion fields, it is given by

$C(t)=\left\langle\operatorname{tr}\left[G_{u}(0, t) \gamma_{5} G_{d}(t, 0) \gamma_{5}\right]\right\rangle$,

where $\langle\ldots\rangle$ means average over the gauge ensemble, the trace $\operatorname{tr}[\ldots]$ is restricted to spin and colour indices only, and we denote by $G_{u}(0, t)$ the propagator for a $u$-quark from 0 to $t$, and correspondingly by $G_{d}$ the similar propagator for the $d$-quark. Here three-space indices are understood as at this stage we need not specify the spatial separation, or equivalently the threemomentum. We can use the identity ${ }^{2} G_{d}(y, z)=\gamma_{5} G_{u}(z, y)^{+} \gamma_{5}$ to relate the connected correlator (12) to propagators from a common source (at time $x_{0}=0$ ) through

$C(t)=\left\langle\operatorname{tr}\left[G_{u}(0, t) G_{u}(0, t)^{+}\right]\right\rangle$.

Thus only propagators for one flavour at one source point are needed for the computation of the charged meson correlator. As we discuss later, it is more efficient, however, to evaluate correlation functions from a wider set of sources.

In Table 1 we give the correspondence between bilinear operators of the form $\bar{d} \Gamma u$, where $\Gamma$ is an hermitian combination of Dirac $\gamma$-matrices, and the mesonic state that is associated with each of them (in the limit $a \rightarrow 0$, i.e. neglecting $\mathrm{O}(a)$ contamination from states of different parity and isospin).

In this table, $X_{1}^{ \pm}$labels an isotriplet state with $J^{P}=0^{+}$, for which there is no experimental candidate. We note that the asso-

\footnotetext{
1 This result essentially follows from the property that, at maximal twist, the order $a$ piece of the Symanzik effective Lagrangian, $a \mathcal{L}_{5}$, is odd under parity and the flavour exchange $u \leftrightarrow d$.

2 Here (with a little abuse of notation) by ${ }^{+}$we mean complex conjugation and transposition with respect to spin-colour indices only, while $y=\left(\mathbf{y}, y_{0}\right)$ and $z=$ $\left(\mathbf{z}, z_{0}\right)$ are the space-time coordinates.
} 
Table 1

\begin{tabular}{ll}
\hline Meson & Operator \\
\hline$\pi^{ \pm}, \pi^{ \pm}, X_{1}^{ \pm}$ & $\bar{d} \gamma_{5} u, \bar{d} \gamma_{0} u, \bar{d} i \gamma_{0} \gamma_{5} u$ \\
$\rho^{ \pm}, \rho^{ \pm}, a_{1}^{ \pm}$ & $\bar{d} i \gamma_{i} \gamma_{0} u, \bar{d} i \gamma_{i} \gamma_{5} u, \bar{d} \gamma_{i} u$ \\
$b_{1}^{ \pm}$ & $\bar{d} i \gamma_{i} \gamma_{0} \gamma_{5} u$ \\
$a_{0}^{ \pm}$ & $\bar{d} u$ \\
\hline
\end{tabular}

ciated operator is in the continuum a component of a conserved current in the theory with two mass degenerate quarks.

We evaluate the two-point (connected) correlators for all the pairs of operators in the same line of Table 1 . In view of the symmetries of the lattice theory at maximal twist [2,3], such correlators are in general non-zero: e.g., the correlator obtained from the insertion of the first (or second) operator in the second line of Table 1 with the third operator in the same line is an $\mathrm{O}(a)$ quantity (in fact $\rho^{ \pm}$and $a_{1}^{ \pm}$carry different continuum quantum numbers). Since we also use a local and extended (fuzzed) source and sink in all cases we consider, we will have either $6 \times 6$ or $2 \times 2$ matrices of correlators available.

Therefore, we measure in general correlation functions of several different pairs of operators $\left(\left\langle O_{\alpha} O_{\beta}\right\rangle\right.$, with $\left.\alpha, \beta=1, \ldots, N\right)$ at source and sink. We then use a factorising fit expression where $i=1, \ldots, M$ states (with energy denoted by $E_{i}$ ) are included

$C_{\alpha \beta}(t)=\sum_{i=1}^{M} c_{\alpha}^{i} c_{\beta}^{i}\left(e^{-E_{i} t} \pm e^{-E_{i}(T-t)}\right)$.

Here $T$ is the lattice temporal extent and the \pm sign is determined by the properties of the chosen operators under time-reflection. By simultaneously fitting $N \times N$ correlators with $M$ states, we can optimally determine energies and couplings. From them we evaluate other quantities of interest, such as $a f_{\pi}$ and $a m_{\text {PCAC. We use }}$ conventional methods to determine the optimal $t$ range, $N$ - and $M$-values to be employed in the fits. We take into account statistical correlations among observables [17] through correlated fits to establish that the $\chi^{2}$ value is acceptable. Our final fitted values are obtained from uncorrelated fits, since that introduces less bias [17], although the $\chi^{2}$ values are smaller than those obtained including correlations. We also checked that the fits are stable when taking into account correlations. For pseudoscalar mesons we use mainly $M=1$ as well as $N=4$ or 6 , and select the minimum value of $t$ such that the effective masses (or energies) from different matrix elements agree.

We conclude by recalling that, owing to reflection invariance of the lattice action (the Euclidean analog of the Minkowski complexconjugation, a symmetry that is preserved by Wilson fermions, either chirally twisted or not, see Ref. [3]), all the correlators that are expectation values of fields with definite reflection properties are either real or purely imaginary, depending on whether the whole field product has even or odd reflection-parity. In particular, the expectation values of multilocal fields with negative spatial parity, which are $\mathrm{O}(a)$ quantities, come out to be purely imaginary if one does not take care of inserting the $i$-factors that are needed to render the multilocal field even (rather than odd) under the reflection.

\subsection{Quark propagators from stochastic sources}

Although it is feasible to use $u$-quark propagators from 12 colour-spin sources (with each source being non-zero only for one colour-spin combination) at one space-time point to evaluate mesonic correlators, it is preferable to use the information contained in the gauge configurations more fully, especially in the case of such CPU-time expensive simulations as dynamical quark simulations. One efficient way to achieve this goal is to use stochastic sources. To keep the noise-to-signal ratio reasonable, it is mandatory to use time-slice sources rather than full volume sources. A great reduction of the noise-to-signal ratio over conventional stochastic methods (see Ref. [18] for a review) can be obtained $[19,20]$ by using the "one-end-trick" which is described below. A similar method, called random wall, was used by MILC [21].

The starting point of all stochastic methods for computing quark propagators is the introduction of random sources, $\xi_{i}^{r}$, where $i=1, \ldots, V_{s}$ spans the set of the source degrees of freedom (colour, spin, space, time) and $r=1, \ldots, R$ labels the noise samples generated for each gauge configuration. The corresponding average satisfies

$\lim _{R \rightarrow \infty}\left[\xi_{i}^{*} \xi_{j}\right]_{R}=\delta_{i j}, \quad \lim _{R \rightarrow \infty}\left[\xi_{i} \xi_{j}\right]_{R}=0$

which can be achieved by various different noise choices, such as $\xi_{i}^{r}=( \pm 1 \pm i) / \sqrt{2}$ or Gaussian (complex) noise.

As a next step, we invert the lattice Dirac matrix $M$ (for one given quark flavour) on each sample of this source,

$\phi_{j}^{r}=M_{j k}^{-1} \xi_{k}^{r}$,

so that averaging over $r$ ( $R$ samples) gives

$\left[\xi_{i}^{r *} \phi_{j}^{r}\right]_{R}=\left[\xi_{i}^{r *} M_{j k}^{-1} \xi_{k}^{r}\right]_{R}=M_{j i}^{-1}+$ noise,

where $j$ can be arbitrary and $i$ belongs to the set of indices for which the source is non-vanishing, which we assume to be of size $V_{s}$. The quantity (17) is an unbiased estimator of the quark propagator from $i$ to $j$. Unfortunately, here the noise is expected to be as $\approx \sqrt{V_{s}} / \sqrt{R}$ whereas the signal is $\approx 1$ at best. Variance reduction is thus very necessary. Furthermore for a meson correlator, the signal behaves as $\exp \left(-m_{\text {meson }} t\right)$ which decreases rapidly with increasing $t$.

The 'one-end-trick' allows $[19,20]$ a more favourable signal-tonoise ratio. Consider the product $\phi_{i}^{r *} \phi_{j}^{r}$ where the stochastic source is now non-zero for all colour-spin indices and all space points at only one time, denoted by $t_{0}$ (time-slice source). Then upon averaging over $r$ one has

$$
\begin{aligned}
{\left[\phi_{i}^{r *} \phi_{j}^{r}\right]_{R} } & =\left[\left(M_{i k}^{-1} \xi_{k}^{r}\right)^{*} M_{j m}^{-1} \xi_{m}^{r}\right]_{R} \\
& =M_{i k}^{-1 *} M_{j k}^{-1}+\text { noise }
\end{aligned}
$$

where the sum over $k$ includes all source components. This quantity is an unbiased estimator for the product of the quark propagators $M_{j k}^{-1} M_{k i}^{-1+}$ from the source to sites $i$ and $j$ on each gauge configuration. Then contracting with $\delta_{i j}$ and summing over space at fixed time-slice $t$ yields the full zero three-momentum ( $\pi^{ \pm}$-channel) correlator from $t_{0}$ to $t$. The noise counting is now more favourable. There are $V_{s}^{2}$ noise terms, which yield a standard deviation of order $V_{s}$, but the signal itself is of order $V_{s}$. This is such big an advantage that it is sufficient to employ just one sample of noise per gauge configuration $(R=1)$. As we discuss below, the optimal way to choose the time-slice $\left(t_{0}\right)$ at which the stochastic source is located, is to change it randomly as the gauge configuration is changed. It should be remarked that the 'one-end-trick', as formulated above, only works for the case of a zero threemomentum interpolating field of the form $\bar{d} \gamma_{5} u$ at the source time $\left(t_{0}\right)$.

A convenient extension of the 'one-end-trick', that allows meson-to-meson correlators with any Dirac structure at the source to be evaluated, requires consideration of four $(\beta=1,2,3,4)$ "linked" sources of the form

$\xi_{\alpha, c, \mathbf{x}, x_{0}}^{\left(\beta ; t_{0}\right)}=\delta_{\alpha \beta} \delta_{x_{0} t_{0}} \eta_{c, \mathbf{x}}$,

where $\alpha$ and $c$ are Dirac and colour indices respectively, while $\eta$ is a non-vanishing noise field. Such sources, which are non-zero 
only on a given time-slice $\left(t_{0}\right)$ and when the Dirac index value equals $\beta$, are called "linked" because they involve a common noise field $\eta .^{3}$ One can check that by replacing $\xi$ and $\xi^{*}$ in the lefthand side of Eq. (18) by two of these linked sources, say $\xi^{\left(\beta ; t_{0}\right)}$ and $\xi^{\left(\gamma ; t_{0}\right) *}$, and choosing appropriately $\beta$ and $\gamma$, it is possible to evaluate the two-point correlators with a field of the form $\bar{d} \Gamma u$ at the source $\left(x_{0}=t_{0}\right)$ with any Dirac matrix $\Gamma$. This very useful extension, which we have thoroughly exploited in the present paper, comes at a moderate price. One must in fact only perform four separate inversions (per gauge configuration and per noise sample), one for each of the four linked sources $\xi^{\left(\beta ; t_{0}\right)}, \beta=1, \ldots, 4$.

To further extend the one-end trick with linked sources to nonzero three-momentum or to spatially non-local mesonic operators is completely straightforward, at the cost of more inversions. One creates further linked sources $F \xi$ (where $F$ denotes a product of links) with the desired spatial properties, and computes the quark propagators originating from them, $\phi_{F}=M^{-1} F \xi$. Combining the latter with the quark propagator stemming from $\xi$, i.e. $\phi=M^{-1} \xi$, yields the product $\phi^{*} \phi_{F}$, from which, upon averaging over the noise, one can evaluate a set of correlators with the meson field $\bar{d} \gamma_{5} F u$ inserted at the source (and all possible spatial structures at the sink). Employing linked sources, as explained above, one can finally evaluate correlators with the meson field $\bar{d} \Gamma F u$ inserted at the source with any spatial structure $F$ and Dirac matrix $\Gamma$, while retaining all advantages of the one-end trick.

In this work we use fuzzing, see Appendix D.2 and Ref. [23], to create spatially non-local meson operators, since this procedure is computationally fast also at the sink. The fuzzed meson source is constructed from a sum of straight paths of length $6 a$, in the six spatial directions, between quark and antiquark. These straight paths are products of fuzzed gauge links. Here for the fuzzed links we use the iterative procedure defined in Appendix D.2 with $\lambda_{s}=$ 0.25 and $n=5$.

In principle one could hope to extend the approach described above to baryonic correlators (choosing $\xi$ as a cubic root of 1 ) but the signal to noise ratio will be less favourable (noise induced standard deviation will be $\approx V_{s}^{3 / 2}$ versus signal $\approx V_{s}$ ). Unfortunately one finds that this extension of the stochastic method to baryons is not any improvement over using point-like sources. In general, the choice of the optimal stochastic methods needs to be investigated on a case by case basis.

\subsection{On the way of choosing the source time-slice}

As discussed above, we invert on spatial-volume stochastic sources located at time $t_{0}$, where $0 \leqslant t_{0}<T$ can be chosen differently for each gauge configuration. We have explored two ways of changing the source time-slice $t_{0}$. One consists in moving $t_{0}$ cyclically through the lattice. This means that we choose $n$ equally spaced values for the source time locations, $t_{0}^{(i)}, 0 \leqslant i<n$. Then we invert on the $j$ th gauge configuration using sources that are nonvanishing only at the time-slices $t_{0}=t_{0}^{(j \bmod n)}$. Hence, we invert from the same time-slice only every $n$ configurations, i.e. after one cycle. Even though this method should decorrelate the measurement on two consecutive gauge configurations better than when the time-slices are kept fixed, it has the drawback that after a relatively short number of configurations the same time-slice is used again. Actually, at least for the mesonic correlators studied in this paper, it turns out that two measurements from the same timeslice, but 8 trajectories apart, are much more correlated than two measurements from different time-slices, but only two trajectories

\footnotetext{
3 Note that "linked" sources are different than "spin-diluted" sources $[18,22]$ since these require different random numbers for each spin.
}

apart. Furthermore the analysis with the $\Gamma$ method of Ref. [24] described in Section 4.1 and Appendix $C$ becomes ill-defined, because translational invariance is broken. This invariance can be recovered, however, by averaging over cycles and using the $\Gamma$ method on the cycle-averaged ensemble.

The second way of moving the time-slice we explored was to choose the value of $t_{0}$ randomly for every gauge configuration we inverted on. This method also maintains translational invariance properly for a large enough configuration ensemble. It is therefore expected to work better than the aforementioned cyclical way. This will indeed turn out to be the case, as we shall see below, where we discuss in more detail the effects of these two ways of generating source time-slices.

\section{Computations in the neutral meson sector}

Lattice QCD with maximally twisted Wilson fermions enjoys the remarkable property that, even if the action is not $\mathrm{O}(a)$ improved, all the physically relevant observables are affected by cutoff effects only at order $a^{2}$ (and higher). Among these $\mathrm{O}\left(a^{2}\right)$ cutoff effects will be a violation of parity and (in part) isospin. Isospin and parity violations have several consequences for meson spectroscopy. For instance, (1) neutral and charged mesons can have different masses, (2) quark-disconnected contributions are needed for neutral isovector mesons and (3) correlators receive contributions from states that in the continuum limit carry different parity and isospin quantum numbers [3]. Here we discuss how we compute the correlators for neutral mesons and, in particular, the quark-disconnected (for brevity called simply "disconnected" below) contributions. We illustrate our approach in the relevant case of the neutral pseudo-scalar meson.

The neutral pion can be created by the operator $\sqrt{2} \bar{\psi} \gamma_{5} \tau_{3} \psi$ which, at maximal twist, in the twisted quark basis reads $(i / \sqrt{2}) \times$ $\bar{\chi} \chi=(i / \sqrt{2})(\bar{u} u+\bar{d} d)$. When this operator is inserted at source and sink, we will have to consider the correlators

$C_{\text {tot }}(t)=\langle(\bar{u} u+\bar{d} d)(t)(\bar{u} u+\bar{d} d)(0)\rangle / 2$,

where again three-space indices are understood. The latter can be rewritten in the form

$C_{\text {tot }}(t)=\widetilde{C}(t)+\widetilde{D}(t)$,

$\widetilde{C}(t)=-\left\langle\operatorname{tr}\left[G_{u}(0, t) G_{u}(t, 0)\right]+\operatorname{tr}\left[G_{d}(0, t) G_{d}(t, 0)\right]\right\rangle / 2$,

$\widetilde{D}(t)=\left\langle\operatorname{tr}\left[G_{u}(0,0)+G_{d}(0,0)\right] \operatorname{tr}\left[\left(G_{u}(t, t)+G_{d}(t, t)\right]\right\rangle / 2\right.$,

with the trace $\operatorname{tr}[\ldots]$ running only over spin and colour indices. As usual, we can relate the connected contribution $(\widetilde{C})$ to propagators from a common source (at time $x_{0}=0$ ) through

$$
\begin{aligned}
\widetilde{C}(t)= & -\left\langle\operatorname{tr}\left[\gamma_{5} G_{u}(0, t) \gamma_{5} G_{d}(0, t)^{+}\right]\right. \\
& \left.+\operatorname{tr}\left[\gamma_{5} G_{d}(0, t) \gamma_{5} G_{u}(0, t)^{+}\right]\right\rangle / 2 .
\end{aligned}
$$

The disconnected contribution can be expressed as

$\widetilde{D}(t)=\left\langle\operatorname{tr}\left[G_{u}(0,0)+G_{u}(0,0)^{+}\right] \operatorname{tr}\left[G_{u}(t, t)+G_{u}(t, t)^{+}\right]\right\rangle / 2$.

Thus we see that to evaluate the correlation (19) we need both $u$ and $d$-quark sources for the connected contribution as well as an evaluation of the disconnected contribution for $u$-quarks at both initial and final $t$-value. This is at variance with the $\pi^{+}$correlator which can be evaluated from a $u$-quark source alone and which has no disconnected contribution. The evaluation of the disconnected loops is detailed in Appendix B, including discussion of both the hopping-parameter method for the reduction of the stochastic noise [25] and a new powerful method of variance reduction applicable in many cases.

In Table 2 we give the correspondence between bilinear operators of the form $\bar{u} \Gamma u \pm \bar{d} \Gamma d$, where $\Gamma$ is an hermitian combination 
Table 2

\begin{tabular}{ll}
\hline Meson & Operator \\
\hline$\pi^{0}, \pi^{0}, f_{0}$ & $\bar{\chi} i \gamma_{0} \gamma_{5} \tau_{3} \chi, \bar{\chi} \chi, \bar{\chi} \gamma_{5} \tau_{3} \chi$ \\
$\eta, \eta, a_{0}^{0}$ & $\bar{\chi} i \gamma_{0} \gamma_{5} \chi, \bar{\chi} \tau_{3} \chi, \bar{\chi} \gamma_{5} \chi$ \\
$\rho^{0}, \rho^{0}, h_{1}$ & $\bar{\chi} \gamma_{i} \tau_{3} \chi, \bar{\chi} i \gamma_{i} \gamma_{0} \gamma_{5} \chi, \bar{\chi} i \gamma_{i} \gamma_{0} \tau_{3} \chi$ \\
$\omega, \omega, b_{1}^{0}$ & $\bar{\chi} \gamma_{i} \chi, \bar{\chi} i \gamma_{i} \gamma_{0} \gamma_{5} \tau_{3} \chi, \bar{\chi} i \gamma_{i} \gamma_{0} \chi$ \\
$a_{1}^{0}$ & $\bar{\chi} i \gamma_{i} \gamma_{5} \tau_{3} \chi$ \\
$f_{1}$ & $\bar{\chi} i \gamma_{i} \gamma_{5} \chi$ \\
$X_{1}^{0}$ & $\bar{\chi} \gamma_{0} \tau_{3} \chi$ \\
$X_{0}^{0}$ & $\bar{\chi} \gamma_{0} \chi$ \\
\hline
\end{tabular}

of $\gamma$-matrices, and the neutral mesonic state that is associated with each of them in the limit $a \rightarrow 0$ (i.e. ignoring $\mathrm{O}(a)$ contaminations from states of different parity and isospin).

Here $X_{1}^{0}\left(X_{0}^{0}\right)$ labels an isotriplet (isosinglet) state with $J^{P C}=$ $0^{+-}$, for which no experimental candidate is known. We remark that these operators are conserved isotriplet (isosinglet) currents in the continuum theory with two mass degenerate quarks.

As in the charged channel, we evaluate the two-point correlators where only pairs of meson operators appearing in the same line of Table 2 above are inserted. Since we use a local and extended (fuzzed) source and sink in each case, we have either $6 \times 6$ or $2 \times 2$ matrices of correlators available. The connected correlators are actually the same for certain states of different isospin (e.g., $\eta$ or $\pi$ ). The same 'one-end-trick' discussed above, based on the use of stochastic time-slice sources with random choice of its position on each gauge configuration, can be used for the connected neutral correlator.

In more detail, we use four "linked" sources $\left(\xi^{(\beta)}\right.$, see Section 2.1) and further four fuzzed sources based on the same noise field $\left(F \xi^{(\beta)}\right)$ to compute ordinary and fuzzed $u$-quark propagators from one time-slice to all points. This set of eight sources is just the same we used to evaluate correlators of charged mesons. For neutral mesons, we inverted the lattice Dirac matrix of the $d$-quark on the same (non-fuzzed) four stochastic sources $\left(\xi^{(\beta)}\right)$ as above and on the corresponding four stochastic sources with the lowest possible three-momentum $(2 \pi / L$, for simplicity taken always in the $x$-direction). In principle mesonic operators with non-zero anisotropic three-momentum have less symmetry then their counterparts with vanishing three-momentum, implying that more correlators may take non-zero values. Here we do not evaluate these additional correlators. We do take care, however, to distinguish between the vector meson correlators with three-momentum parallel to spin and those with three-momentum perpendicular to it. As shown elsewhere [26], a study of the difference between these correlators can shed some light on the mixing of $\rho$ mesons with their decay products $(\pi \pi)$.

\section{Simulation algorithm and error analysis}

In this section we provide details on the algorithms we used to generate the gauge configurations and information on the methods employed for the estimate of statistical errors.

In Table 3 we give the list of the key parameters characterising the simulations we are going to use in this paper. All simulations $B_{1}-B_{5}$ have been performed at a fixed value of the gauge coupling $\beta=3.9$ and a fixed value of the hopping parameter $\kappa=\left(8+2 a m_{0}\right)^{-1}=0.160856$ on $24^{3} \times 48$ lattices. In addition to the values of $a \mu_{q}$ we provide in Table 3 the number of trajectories, $N_{\text {traj }}$, produced after allowing for 1500 equilibration trajectories, and the number of gauge configurations, $N_{\text {cfg }}$, that were saved on disk (one every second trajectory). For every value of $a \mu_{q}$ we have reached $\sim 5000$ equilibrated trajectories.
Table 3

Summary of all simulation points

\begin{tabular}{lllrr}
\hline Run & $L^{3} \times T$ & $a \mu_{q}$ & $N_{\text {traj }}$ & \multicolumn{1}{c}{$N_{\text {cfg }}$} \\
\hline$B_{1 a}$ & $24^{3} \times 48$ & 0.0040 & 5000 & 2500 \\
$B_{1 b}$ & 0.0040 & 1341 & 670 \\
$B_{1 c}$ & & 0.0040 & 3380 & 1690 \\
$B_{2}$ & & 0.0064 & 5192 & 2500 \\
$B_{3 a}$ & 0.0085 & 3753 & 1876 \\
$B_{3 b}$ & 0.0085 & 940 & 470 \\
$B_{4}$ & & 0.0100 & 5000 & 2500 \\
$B_{5 a, b}$ & & 0.0150 & 2500 & 1250 \\
\hline
\end{tabular}

We give the lattice size $L^{3} \times T$ and the value of the twisted mass $a \mu_{q}$. In the last two columns we quote the number of equilibrated trajectories $N_{\text {traj }}$ produced and the number of configurations $N_{\text {cfg }}$ saved to disk and finally stored within ILDG, see the review [27] for further links and references. All runs listed in the table have been performed at $\beta=3.9$ and $\kappa=0.160856$.

\section{Table 4}

HMC algorithm parameters

\begin{tabular}{llllllll}
\hline Run & Int. & $N_{0,1,2}$ & $\tilde{\mu}_{1}$ & $\lambda_{0,1,2}$ & $N_{1,2}^{\text {csg }}$ & $P_{\text {acc }}$ & $\tau_{\text {int }}(P)$ \\
\hline$B_{1 a, b}$ & $2 \mathrm{MNp}$ & $2,3,6$ & 0.018 & $0.19,0.20,0.21$ & 0,0 & 0.85 & $47(15)$ \\
$B_{1 c}$ & SW & $2,3,6$ & 0.018 & - & 0,0 & 0.90 & $43(15)$ \\
$B_{2}$ & $2 \mathrm{MNp}$ & $2,3,6$ & 0.025 & $0.19,0.20,0.21$ & 0,0 & 0.90 & $23(7)$ \\
$B_{3 a, b}$ & $2 \mathrm{MN}$ & $2,3,5$ & 0.020 & $0.19,0.20,0.21$ & 7,1 & 0.90 & $13(3)$ \\
$B_{4}$ & $2 \mathrm{MNp}$ & $2,3,6$ & 0.035 & $0.19,0.20,0.21$ & 0,0 & 0.90 & $15(4)$ \\
$B_{5 a, b}$ & SW & $2,2,6$ & 0.050 & - & 0,0 & 0.90 & $30(8)$ \\
\hline
\end{tabular}

For all ensembles we specify the integration scheme, the number of time steps on each time scale $N_{0,1,2}$, the precondition mass $\tilde{\mu}_{1}=2 \kappa \mu_{1}$, the $\lambda$-values for the $2 \mathrm{MN}$ integration scheme, the number of saved solutions $N_{\text {csg }}$ for the chronological solver guess, the acceptance rate $P_{\text {acc }}$ observed in the run and the integrated autocorrelation time of the plaquette $\tau_{\text {int }}(P)$. The trajectory length was set to $\tau=1 / 2$ for all runs and we used always $N_{\mathrm{PF}}=2$ pseudo-fermion fields.

In case we have several ensembles (as for instance for $B_{1}$ ) or several replicas (as for instance for $B_{5}$ ) for the same lattice parameter set, we denote this by adding an extra subscript, $a, b, \ldots$. For our smallest value $a \mu_{q}=0.004$ we extended our statistic from about 5000 trajectories (ensemble $B_{1 a}$ ) to $\sim 10000$ trajectories (if also trajectories from ensembles $B_{1 b}$ and $B_{1 c}$ are counted).

The algorithm we used is a HMC algorithm [28] with mass preconditioning $[29,30]$ and multiple time scale integration, as described in detail in Refs. [31,32]. The algorithm parameters we employed for the various runs can be found in Table 4, where we mostly follow the notation of Ref. [31]. The integration schemes we used are the Sexton-Weingarten (SW) scheme [33], the second order minimal norm scheme (2MN) [34] and its position version (2MNp). We also list the number of integration steps $N_{i}$ for timescale $i$ (for details see Ref. [31]). We recall that $N_{2}$ represents the number of integration steps of the outermost (largest) timescale. Thus the number of integration steps of the smallest (i.e. innermost) time-scale (the one referring to the gauge field integration) is given by $N_{2} \cdot N_{1} \cdot N_{0}$. The preconditioning mass is given by $\tilde{\mu}_{1}=2 \kappa \mu_{1}$, with $\mu_{1}$ typically larger than $\mu_{q}$ by a factor $\mathrm{O}(10)$.

The second order minimal norm integration scheme on timescale $i$ is parametrised by one real number, $\lambda_{i}$. We also give the number, $N_{i}^{\mathrm{csg}}$, of solutions of the Dirac equation we save for the chronological solver guess [35] with the purpose of evaluating the two force terms $(i=1,2)$ associated to pseudo-fermion integration ( $i=0$ refers to the pure gauge force). The notation $N_{1,2}^{\text {csg }}=0$ means that no chronological solver guess was used there. Finally, we quote the acceptance rate $P_{\text {acc }}$ observed in the simulation and the integrated autocorrelation time $\tau_{\text {int }}(P)$ of the plaquette expectation value. The trajectory length was set to $\tau=1 / 2$ in all our runs and we always used $N_{\mathrm{PF}}=2$ pseudo-fermion fields. For details on the linear solvers we employed to invert the Dirac matrix we refer to Ref. [36].

To give guidance on the computational cost of such simulations, we specify the resource used at our lightest $\mu_{q}$-value where the 
CG iterations for one trajectory cost about 115 Tflop. The production of 5000 trajectories amounted to about 17 rack days on the BlueGene/L installation in Jülich, with our code running with an efficiency of about $18 \%$ for the $B_{1}$ parameter set.

\subsection{Statistical error analysis}

A reliable estimate of the statistical errors on the measured quantities is extremely important for many reasons. We discuss here only the points which are of special relevance in our analysis. If the basic systematic effects in the lattice simulation, originating from the lattice discretisation, the finite volume and the mass of the dynamical quarks are to be addressed, the statistical accuracy on all the relevant quantities has to be understood very well. In fact, on the one hand, relevant but tiny systematic effects can only be detected with high statistical accuracy, on the other hand underestimated statistical errors can artificially increase the significance of systematic effects. The PCAC quark mass, though not a physical quantity, plays here a special role, since the precision by which it is set to zero is related to the accuracy (see Sections 1.2 and 1.3) by which we can expect to be at maximal twist. Secondly, small statistical errors in low-energy hadronic quantities is an expected virtue of the twisted mass formulation, where a sharp infra-red cut-off ensures a stable MC evolution of the lattice system. Of course we have to make sure that an apparently small statistical error does not come as a result of large unnoticed autocorrelations in the MC history. So autocorrelations in the measured quantities must be accurately analysed. Finally, a detailed analysis of the statistical errors delivers as a by-product the integrated autocorrelation time $\tau_{\text {int }}$ of the studied observable, from which the efficiency of the employed algorithm as a function of the simulation parameters can be quantified (see Section 4.2).

Given the importance of getting a reliable estimate of statistical errors, results have been cross-checked using different approaches. As for the estimate of autocorrelation times two different kinds of analyses have been performed: one based on a standard datablocking (or binning) procedure and another one relying on the so-called $\Gamma$-method [24,37]. In order to keep self-contained this paper we discuss these methods in some detail in Appendix C. Since there are arguments [24] supporting the superiority of the $\Gamma$-method over data-blocking, the former will be our method of choice in the evaluation of $\tau_{\text {int }}$ and the error on it, for several observables. In particular the $\Gamma$-method has been used to estimate the statistical error on the plaquette and $m_{\mathrm{PCAC}}$, which turn out to have large autocorrelation times. For all the other observables having significantly smaller autocorrelation times data-blocking and $\Gamma$-method typically give quite similar error estimates.

Cross-correlations among different observables are properly taken into account in our error analysis by using standard jackknife or bootstrap [38] or performing fits based on a definition of $\chi^{2}$ that involves the inverse covariance matrix (see Eq. (42) and the discussion of Method A in Section 7.1).

\subsection{Autocorrelation times}

For a primary observable 0 , i.e. one that can be viewed as a linear combination of expectation values of multi-local operators, the integrated autocorrelation time is in principle given by

$\tau_{\text {int }}(0)=\frac{1}{2}+\sum_{n=1}^{\infty} \frac{\Gamma_{O}(n)}{\Gamma_{O}(0)}$,

where $\Gamma_{0}(n)$ is the autocorrelation function of the observable $O$ (see Eq. (C.2)). The autocorrelation times for the plaquette and fermionic quantities, like $a m_{\mathrm{PS}}, a f_{\mathrm{PS}}$ and $a m_{\mathrm{PCAC}}$, were determined using the $\Gamma$-method as described in Ref. [24] (see also Appendix C). This method allows the determination of $\tau_{\text {int }}$ also
Table 5

Estimated integrated autocorrelation times for $a m_{\mathrm{PS}}, a f_{\mathrm{PS}}$ and $a m_{\mathrm{PCAC}}$

\begin{tabular}{llll}
\hline Run & $\tau_{\text {int }}\left(a m_{\mathrm{PS}}\right)$ & $\tau_{\text {int }}\left(a f_{\mathrm{PS}}\right)$ & $\tau_{\text {int }}\left(a m_{\mathrm{PCAC}}\right)$ \\
\hline$B_{1 a}^{\text {cyc }}$ & $7(1)$ & $13(4)$ & $60(24)$ \\
$B_{1 a}^{\text {rnd }}$ & $6.6(1.1)$ & $8(1)$ & $20(5)$ \\
$B_{1 a, b, c}^{\text {rnd }}$ & $5.9(7)$ & $7(1)$ & $23(5)$ \\
$B_{2}^{\text {cyc }}$ & $17(4)$ & $33(8)$ & $43(14)$ \\
$B_{3}^{\text {cyc }}$ & $10(2)$ & $11(2)$ & $66(27)$ \\
$B_{4}^{\text {cyc }}$ & $7(2)$ & $14(4)$ & $54(23)$ \\
$B_{5 a, b}^{\text {cyc }}$ & $20(6)$ & $14(3)$ & $105(51)$ \\
\hline
\end{tabular}

The labels cyc and rnd refer to the cyclic and random choice of the source, see text. All integrated autocorrelation times are given in units of trajectories of length $1 / 2$. The fact that for the ensemble $B_{5}^{\text {cyc }}$ we find a rather large autocorrelation time with, however, a large error we attribute to the usage of 2 replica in the analysis.

\section{Table 6}

Measurement methods for fermionic quantities

\begin{tabular}{llrll}
\hline Run & Method & $t_{p}$ & Time-slices & $\chi^{2} /$ d.o.f. \\
\hline$B_{1 a}$ & cyclic & 8 & $0,12,24,36$ & $0.12 / 39$ \\
$B_{1 a, b, c}$ & random & 10 & - & $2.50 / 39$ \\
$B_{2}$ & cyclic & 16 & $0,6,12,18,24,30,36,42$ & $1.15 / 39$ \\
$B_{3}$ & cyclic & 8 & $0,12,24,36$ & $2.38 / 39$ \\
$B_{4}$ & cyclic & 8 & $0,12,24,36$ & $1.85 / 39$ \\
$B_{5 a, b}$ & cyclic & 8 & $0,12,24,36$ & $0.99 / 39$ \\
\hline
\end{tabular}

The source time-slices are either chosen in a cyclic way or randomly. For the cyclic way, $t_{p}$ denotes the number of trajectories between two configurations for which the same time-slice was used. For the random way, it specifies the number of trajectories between two measured configurations. In the cyclic case we also specify the time-slices where the source was located. Finally, the fit range we chose to determine $a f_{\mathrm{PS}}$ and $a m_{\mathrm{PS}}$ was always $10-23$ and the corresponding values of $\chi^{2} /$ d.o.f. for a $2 \times 2$ factorising fit (see Eq. (14)) are quoted in the last column.

for non-primary quantities, as the aforementioned fermionic observables. The values for the plaquette integrated autocorrelation time are collected in Table 4 , those for $a m_{\mathrm{PS}}, a f_{\mathrm{PS}}$ and $a m_{\mathrm{PCAC}}$ in Table 5. All quoted values are given in units of trajectories of length $1 / 2$.

In the case of the ensemble $B_{1 a}$ we employed the two ways of moving the stochastic source through the lattice described in Section 2.2. As can be seen in Table 5, indeed the random way performs better. This is especially significant for $a_{\mathrm{PCAC}}$, for which we observe the longest autocorrelation time among the fermionic quantities. For $a m_{\mathrm{PS}}$ and $a f_{\mathrm{PS}}$ the difference between the two methods is not significant. The somewhat larger autocorrelation time of $B_{2}^{\text {cyc }}$, in particular for $m_{\mathrm{PS}}$ and $f_{\mathrm{PS}}$, stems presumably from the fact that the time slice sources were chosen closer to each other than at the other ensembles.

Table 6 gives details on the computational methods employed to extract the various fermionic quantities. In the case where the random way of moving the source is used, the value of $t_{p}$ reported there represents the number of trajectories (of length $1 / 2$ ) between two consecutively measured gauge configurations. We also give in this table the value of $\chi^{2} /$ d.o.f. obtained when the charged pion correlators (for Euclidean time separations in the range $10 \leqslant t / a \leqslant 23$ ) are fitted using the Ansatz (14).

Looking at the three fermionic observables reported in Table 5, we observe that the integrated autocorrelation times of am ${ }_{\mathrm{PCAC}}$ are significantly larger than those of $a m_{\mathrm{PS}}$ and $a f_{\mathrm{PS}}$. We attribute the large value of the autocorrelation time of am $\mathrm{PCAC}$ to the peculiar phase structure of twisted mass lattice QCD with Wilson type quarks as discussed in Ref. [7]. The simulated values of $\mu_{q}$ are not in a region where the phase transition occurs. However, the system may still feel the presence of this phase transition. The situation is similar for the plaquette value, as also discussed in Ref. [7], and indeed the integrated autocorrelation times for the plaquette and $a m_{\text {PCAC }}$ are rather similar.

We show the Monte Carlo (MC) history of an estimator of $a m_{\mathrm{PCAC}}$, see Eq. (32), for our lightest quark mass $\left(a \mu_{q}=0.0040\right)$ 


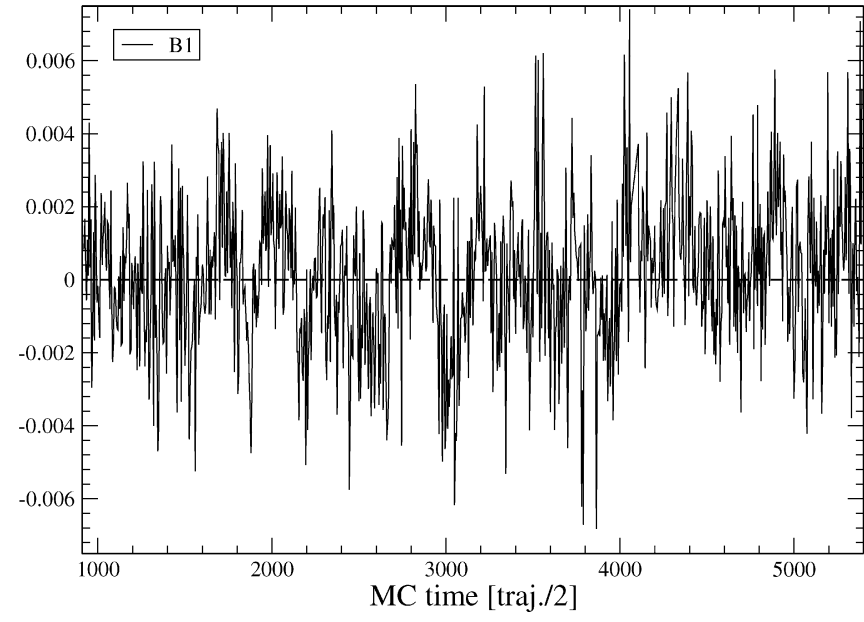

Fig. 1. The Monte Carlo history of the ratio of correlators defining the PCAC quark mass estimator described in the text on the configurations of the ensemble $B_{1}$. The configuration number corresponds to the number of trajectories divided by two.

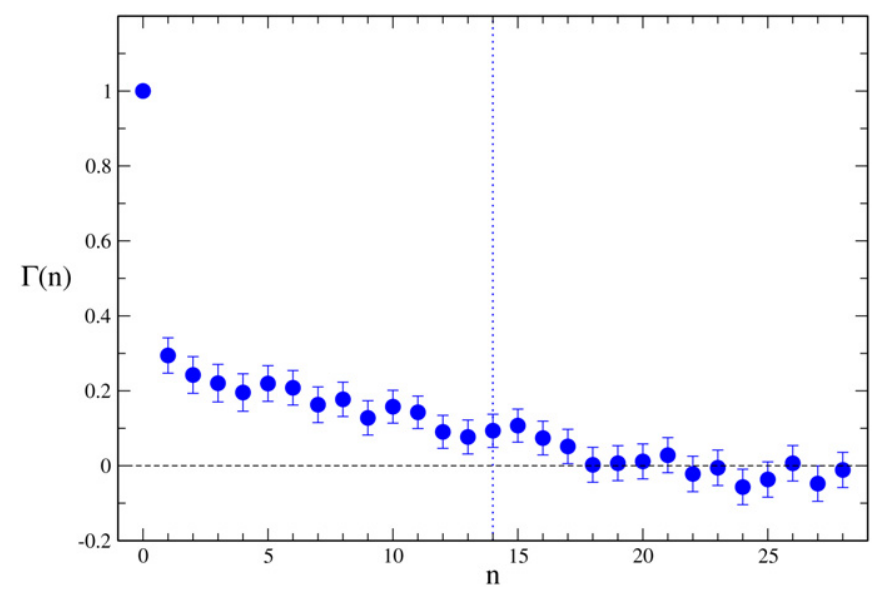

Fig. 2. The autocorrelation function (see Eq. (C.2)) from the data presented in Fig. 1. The vertical line shows the window $W$ from Eq. (C.3) used to evaluate the integrated autocorrelation function.

in Fig. 1. More precisely, we plot for each gauge configuration the axial-pseudoscalar correlator at $t / a=10$ (where the pion ground state is dominant) multiplied by the factor $0.5 \mathrm{am}_{\mathrm{PS}} / C_{\mathrm{PP}}(10)$, where the average over all gauge configurations is used in $C_{\mathrm{PP}}(10) .{ }^{4}$ The plot in Fig. 1 shows long-ranged fluctuations in $\mathrm{MC}$ time. The autocorrelation function in Eq. (C.2) from this data set is reported in Fig. 2.

From it an integrated autocorrelation equal to 32(9) trajectories is obtained, in agreement with the result quoted in Table 5 (third line).

Concerning the neutral pseudoscalar meson, a study of the corresponding correlators indicates that the autocorrelations are definitely shorter than 100 (length $1 / 2$ ) trajectories. Thus our error estimates, coming from a bootstrap analysis on blocked data with blocks made of measurements taken from 80 trajectories, are expected to be reliable.

Within the relatively large errors of our estimates of the autocorrelation times, it is actually not possible to find a significant dependence on the value of the twisted mass $a \mu_{q}$ for any of the fermionic quantities discussed here.

\footnotetext{
4 Note that the average of this quantity over all gauges is not our best estimator of $a m_{\mathrm{PCAC}}$, since it does not exploit the possibility of averaging over $t$ (the Euclidean time separation).
}

\section{The scale from the static potential}

A convenient way to set the scale in lattice simulations is through measurements of the static potential and the associated hadronic scale $r_{0}$ [39]. Although we will finally not use $r_{0}$ to set the scale in our dynamical simulations, we will use it for a scaling analysis towards the continuum limit and its reliable determination is therefore important for us. The scale $r_{0}$ is defined via the force between static quarks at intermediate distance

$r_{0}^{2} F\left(r_{0}\right)=1.65$,

where numerical calculations are most reliable and hence are expected to lead to very accurate results. We measure the static quark-antiquark potential by determining expectation values of Wilson loops of size $r \times t$ on our ensembles of configurations. Unfortunately, the relative errors of the Wilson loop expectation values increase exponentially with the temporal extension $t$. To reduce these statistical fluctuations one can employ improved static actions amounting to use modified temporal links for building Wilson loops. ${ }^{5}$ However, it is also important to enhance the overlap with the physical ground state of the static system and this can be achieved by invoking iterative spatial smearing techniques together with a variational method to extract the ground state. The computational details for calculating the static potential are given in Appendix D while in the following we want to concentrate on analysis details and physical results.

\subsection{Analysis details and results}

In order to extract the physical scale through Eq. (26) we need an interpolation of the potential and correspondingly of the force between the quarks for arbitrary distances $r$. This interpolation is achieved by fitting the form of $V(r)$ with the ansatz ${ }^{6}$

$V(r)=V_{0}+\frac{\alpha}{r}+\sigma r$.

We employ a two step procedure to perform the interpolation. First we extract the values of the potential $V(r)$ for each $r$ separately using standard variational techniques. In a second step we fit directly the potential ansatz in Eq. (27) to the Wilson loop correlators taking into account all spatial and temporal cross-correlations in the data. These two steps are now described in more detail (see also [41]).

We use five spatial smearing levels $\mathcal{S}_{n} U, n=8,16,24,32,40$, and hence we end up measuring a $5 \times 5$ correlation matrix $C(r, t)$ of spatially smeared and temporally improved Wilson loops (see Appendix D). The variational method results in a linear combination of the string operators, which projects sufficiently well to the ground state of the string, i.e. has the effect of eliminating the closest excited string states. This is done by solving the generalised eigenvalue problem

$C\left(r, t_{1}\right) v_{i}=\lambda_{i}\left(r ; t_{0}, t_{1}\right) C\left(r, t_{0}\right) v_{i}, \quad \lambda_{1} \geqslant \cdots \geqslant \lambda_{5}$,

with $t_{0}=3 a$ and $t_{1}=4 a$ and projecting the correlation matrix to the eigenspace corresponding to the largest eigenvalue, i.e. the ground state,

$\bar{C}(r, t)=\left(v_{1}, C(r, t) v_{1}\right)$.

Based on effective masses and on a $\chi^{2}$-test which takes the temporal cross-correlations between $\bar{C}(r, t)$ and $\bar{C}\left(r, t^{\prime}\right)$ into account, we choose a plateau region from $t_{\min }$ to $t_{\max }$. Too small $t$ values distort the results due to contamination of excited states, while too

\footnotetext{
5 See Ref. [40] for a first use of this idea.

6 Note that we do not use tree level improved distances.
} 

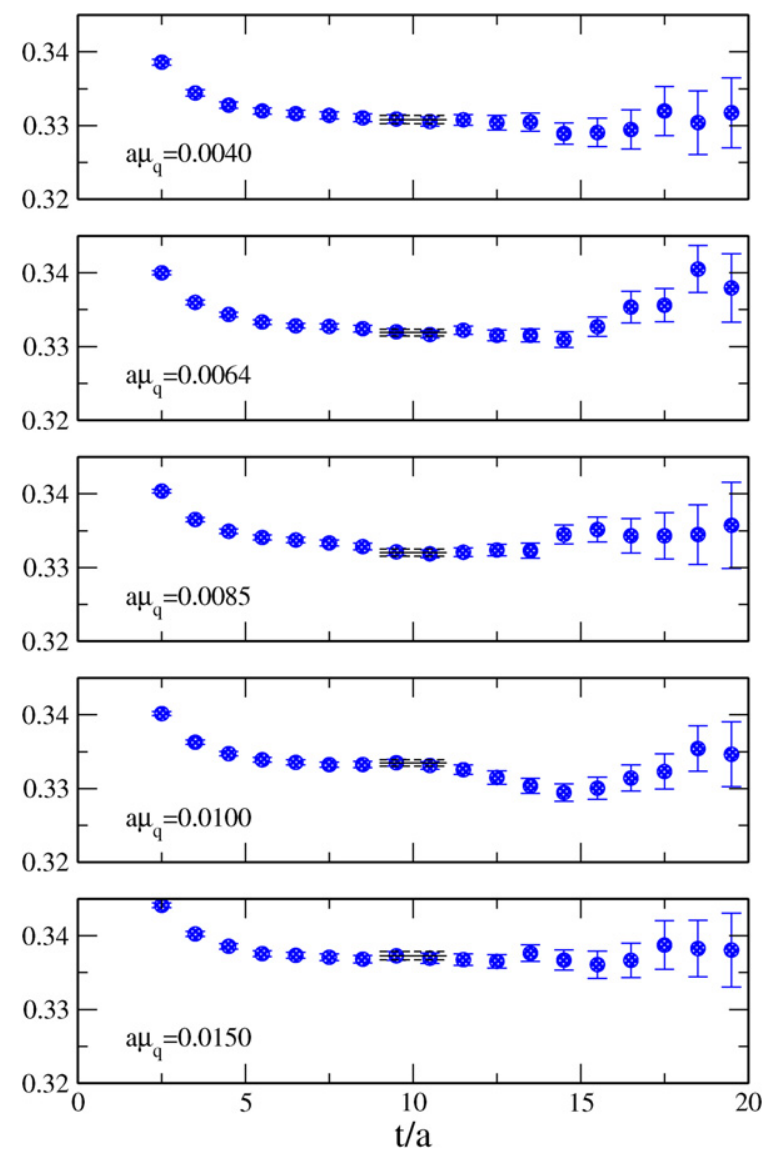

Fig. 3. Effective masses for the ground state energy of the Wilson loop at quarkantiquark separation $r / a=4$, for ensembles $B_{1}$ to $B_{5}$.

Table 7

Fit parameters for the Wilson loop correlators for run $B_{1}$

\begin{tabular}{lllll}
\hline$r / a$ & $t_{\min }$ & $t_{\max }$ & $a V(r)$ & $\chi^{2} /$ d.o.f. \\
\hline 4 & 9 & 11 & $0.3308(10)$ & 1.09 \\
5 & 8 & 10 & $0.3974(15)$ & 0.09 \\
6 & 7 & 9 & $0.4577(26)$ & 2.48 \\
7 & 7 & 9 & $0.5126(31)$ & 1.60 \\
\hline
\end{tabular}

Table 8

Fit parameters for the Wilson loop correlators for run $B_{2}$

\begin{tabular}{lllll}
\hline$r / a$ & $t_{\min }$ & $t_{\max }$ & $a V(r)$ & $\chi^{2} /$ d.o.f. \\
\hline 4 & 9 & 11 & $0.3319(07)$ & 1.56 \\
5 & 8 & 10 & $0.3994(09)$ & 3.27 \\
6 & 7 & 9 & $0.4589(12)$ & 0.16 \\
7 & 7 & 9 & $0.5129(21)$ & 0.13 \\
\hline
\end{tabular}

large values introduce noise. Examples of effective mass plateaus and the chosen fit ranges are provided in Fig. 3 for the Wilson loop correlators of the five ensembles $B_{1, \ldots, 5}$ at quark-antiquark separation $r / a=4$.

The results of our fits are collected in Tables 7-11 where we list the plateau regions (fit range), the values of the extracted potential, $V(r)$, and the $\chi^{2}$ per degree of freedom. The uncertainties in the extracted values of $V(r)$ are calculated using a non-parametric bootstrap method [38].

This first step allows to determine for each $r$ the value of the potential $V(r)$. A straightforward strategy to evaluate the scale $r_{0} / a$ is then to fit the numbers so obtained with the ansatz (27). However, one can diminish the errors on the fit parameters by exploiting the fact that data at different values of $r$ are correlated.
Table 9

Fit parameters for the Wilson loop correlators for run $B_{3}$

\begin{tabular}{lllll}
\hline$r / a$ & $t_{\min }$ & $t_{\max }$ & $a V(r)$ & $\chi^{2} /$ d.o.f. \\
\hline 4 & 9 & 11 & $0.3321(08)$ & 0.75 \\
5 & 8 & 10 & $0.4002(11)$ & 4.39 \\
6 & 7 & 9 & $0.4617(17)$ & 0.40 \\
7 & 7 & 9 & $0.5177(22)$ & 0.02 \\
\hline
\end{tabular}

Table 10

Fit parameters for the Wilson loop correlators for run $B_{4}$

\begin{tabular}{lllll}
\hline$r / a$ & $t_{\min }$ & $t_{\max }$ & $a V(r)$ & $\chi^{2} /$ d.o.f. \\
\hline 4 & 9 & 11 & $0.3335(06)$ & 1.40 \\
5 & 8 & 10 & $0.4013(12)$ & 0.41 \\
6 & 7 & 9 & $0.4616(15)$ & 0.37 \\
7 & 7 & 9 & $0.5177(25)$ & 0.98 \\
\hline
\end{tabular}

Table 11

Fit parameters for the Wilson loop correlators for run $B_{5}$

\begin{tabular}{lllll}
\hline$r / a$ & $t_{\min }$ & $t_{\max }$ & $a V(r)$ & $\chi^{2} /$ d.o.f. \\
\hline 4 & 9 & 11 & $0.3373(07)$ & 1.01 \\
5 & 8 & 10 & $0.4062(08)$ & 3.21 \\
6 & 7 & 9 & $0.4692(14)$ & 1.24 \\
7 & 7 & 9 & $0.5272(22)$ & 2.28 \\
\hline
\end{tabular}

Table 12

Results of the fits for the scale $r_{0} / a$ from the static potential

\begin{tabular}{lllll}
\hline Run & $N_{\text {meas }}$ & d.o.f. & $\chi^{2} /$ d.o.f. & $r_{0} / a$ \\
\hline$B_{1}$ & 625 & 5 & 1.44 & $5.196(28)$ \\
$B_{2}$ & 695 & 5 & 1.80 & $5.216(27)$ \\
$B_{3}$ & 598 & 5 & 2.58 & $5.130(28)$ \\
$B_{4}$ & 602 & 5 & 0.57 & $5.143(25)$ \\
$B_{5}$ & 645 & 5 & 1.92 & $5.038(24)$ \\
\hline
\end{tabular}

The fit range was always $r / a=4-7$. The number of measurements, $N_{\text {meas }}$, and $\chi^{2} /$ d.o.f. are also reported.

Therefore, in a second step we use the ground state projected correlator $\bar{C}(r, t)$ to estimate the covariance matrix

$\operatorname{Cov}\left(r, t ; r^{\prime}, t^{\prime}\right) \equiv\left\langle\bar{C}(r, t) \bar{C}\left(r^{\prime}, t^{\prime}\right)\right\rangle-\langle\bar{C}(r, t)\rangle\left\langle\bar{C}\left(r^{\prime}, t^{\prime}\right)\right\rangle$

from the bootstrap samples of $\bar{C}(r, t)$ and use $\operatorname{Cov}\left(r, t ; r^{\prime}, t^{\prime}\right)$ to construct the $\chi^{2}$ function (see the discussion in Section 7.1). The $r$ and $t$ dependence of $\bar{C}(r, t)$ is fitted with the formula (see Eq. (27))

$$
\begin{aligned}
\bar{C}(r, t) & \sim Z(r) \exp [-t V(r)] \\
& =Z(r) \exp \left[-t\left(V_{0}+\alpha / r+\sigma r\right)\right] .
\end{aligned}
$$

For the temporal fit interval we use the fit ranges $t_{\min }(r)$ to $t_{\max }(r)$ determined in the first step. The fit range in $r$ is chosen so as to include only a few values of $r$ closest to $r_{0}$ in order to minimise both the statistical error and the systematic error coming from the choice of the interpolation formula. Once the best fit parameters $\left(V_{0}, \alpha\right.$ and $\sigma$ ) in Eq. (30) are found, the value of $r_{0} / a$ is obtained straightforwardly by computing the static force from the derivative with respect to $r$ of Eq. (27) and imposing the condition (26) that defines $r_{0}$.

A compilation of the results of our fits is provided in Table 12 where we give the number of measurements $N_{\text {meas }}$ and the $\chi^{2}$ per degree of freedom, in addition to the results for $r_{0} / a$. The final error on $r_{0} / a$ is estimated through jackknife and bootstrap procedures using binning to take residual autocorrelations into account. In Table 12 we give the errors from the jackknife procedure using a binning factor equal to 4 . 


\subsection{Discussion}

There are several sources of systematic effects which can distort a precise and accurate determination of the scale $r_{0} / a$. Here we would like to discuss a few checks that we have performed in order to asses these systematic effects and some procedures to minimise their influence.

\subsubsection{Excited states}

First of all there are contaminations of the ground state energy of the Wilson loops from excited states. We expect that these should be eliminated by our variational calculation of the ground state and our choice of the fit range in $t$, and we have carefully checked the stability of the results under variation of the fit parameters (see Fig. 4). In particular we have checked that we can resolve the first excited state and that the ground state energy remains stable under this procedure. Moreover we have also checked the stability of the ground state under a truncation of the variational operator basis. We would also like to point out that the fit ranges in $t$ were not chosen independently for each value of $\mu_{q}$ and $r$, rather we chose them after taking a global view of the effective mass data for all values of $\mu_{q}$ at given fixed $r$ (see Fig. 3 for the case $r=4 a$ ). This procedure makes sense since the $\mu_{q}$-dependence of the Wilson loop correlators is expected to be rather weak (see below) and is particularly useful in cases where the choice of the fit range for the effective masses cannot be determined unambiguously given the available statistics. Finally we note that contaminations from excited states tend to increase the potential energies and the effect will be more pronounced for the larger Wilson loops. As a consequence, residual contributions from excited states will tend to decrease the value of $r_{0} / a$.

\subsubsection{Interpolation error}

The interpolation of the potential (or the force) as a function of $r$ is not unique. Here we would like to emphasise that we use Eq. (27) only locally as a simple interpolation ansatz without attaching to it any special physical meaning. As a check of this interpolation ansatz, one can use separately the matrices of correlators computed for $r / a=4-6$ and for 5-7 to obtain two different determinations of $r_{0} / a$. Their difference then provides an estimate of the error coming from the interpolation procedure. It turns out that our choice of the fit range $r / a=4-7$ covers this spread typically within 1-2 standard deviations of our final result (see Fig. 4).

\subsubsection{Correlations}

We have already pointed out that it is important to take both the spatial and temporal cross-correlations of the Wilson loop operators into account when fitting them to the ansatz (30). Our finite statistics limits ourselves to short fit ranges in order to obtain a stable covariance matrix, and this is one of the motivations for the rather narrow fit ranges in $t$ in Tables 7-11. In order to assess the effect arising from Wilson loop autocorrelations, we form bins of the data of various sizes, though this reduces the amount of data available for estimating the covariance matrix even further. In fact, it turns out that the fits become unreliable beyond bin size 4 and before the binning error becomes stable. As a consequence we cannot exclude that the errors on $r_{0} / a$ are somewhat underestimated due to residual autocorrelations.

\subsubsection{Mass dependence}

Our results for $\left(r_{0} / a\right)$ are plotted in Fig. 4 . We note that the $a \mu_{q}$ dependence appears to be rather weak, and hence we expect the data for the (purely gluonic) observable $r_{0} / a\left(a \mu_{q}\right)$ to be well described by polynomials of low order in $a \mu_{q}$. In Table 13 we collect the results obtained by fitting our data at different values of $a \mu_{q}$ (see Table 12) to few simple functional forms, namely

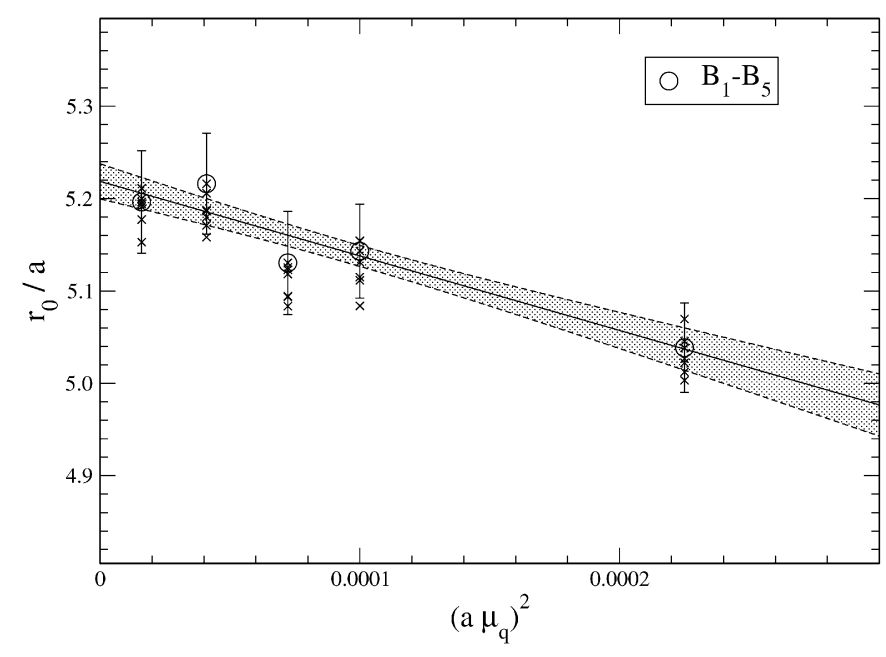

Fig. 4. Mass dependence of $r_{0} / a$. The shaded area shows the error band of the quadratic fit (full line) to the data (circles). The additional plus symbols are further determinations of $r_{0} / a$ corresponding to different values of the fit parameters to the ansatz (30). The spread provides an indication of the systematic error due to interpolation (see text) in $r_{0} / a$.

\section{Table 13}

Results of the fits of the $a \mu_{q}$ dependence of $r_{0} / a$ according to the ansatz (I), (II) and (III) in the text

\begin{tabular}{lllll}
\hline$r_{0} / a$ & $c_{1} \times 10^{-2}$ & $c_{2} \times 10^{-4}$ & Fit range & $\chi^{2} /$ d.o.f. \\
\hline $5.22(2)$ & - & $-0.08(2)$ & $B_{1}-B_{5}$ & 0.85 \\
$5.22(3)$ & - & $-0.09(4)$ & $B_{1}-B_{4}$ & 1.26 \\
$5.28(3)$ & $-0.16(3)$ & - & $B_{1}-B_{5}$ & 1.10 \\
$5.26(5)$ & $-0.12(6)$ & - & $B_{1}-B_{4}$ & 1.37 \\
$5.22(8)$ & $-0.01(18)$ & $-0.08(9)$ & $B_{1}-B_{5}$ & 1.28 \\
\hline
\end{tabular}

(I) $r_{0} / a+c_{2}\left(a \mu_{q}\right)^{2}$,

(II) $r_{0} / a+c_{1}\left(a \mu_{q}\right)$,

(III) $r_{0} / a+c_{1}\left(a \mu_{q}\right)+c_{2}\left(a \mu_{q}\right)^{2}$.

The ansatz (I) is inspired by the fact that with maximally twisted (unlike the case of untwisted) Wilson quarks the lattice fermionic determinant of the $N_{f}=2$ theory depends only quadratically on the bare quark mass. A weaker dependence on the bare quark mass can only appear via the effects of spontaneous chiral symmetry breaking on the static quark potential and would actually be a dependence ${ }^{7}$ on $\left|a \mu_{q}\right|$. This is the motivation for the fit ansatz (II), if it can be assumed that $a \mu_{q}$ is sufficiently small to make the $\left(a \mu_{q}\right)^{2}$-dependence negligible, and (III), if the $\left(a \mu_{q}\right)^{2}$-dependence is instead statistically significant.

The fit based on the ansatz (I) describes our data rather well, as shown in Fig. 4, suggesting that possible effects of spontaneous chiral symmetry breaking in the static potential at distances around $0.5 \mathrm{fm}$ are negligible within our statistical errors. This interpretation is supported also by the other two fits: even if a $\mu_{q}$-dependence of the type (II) cannot be ruled out completely, we observe that not only the $\chi^{2} /$ d.o.f. of the fit (I) compared to (II) is better, but also the best-fit values of $c_{2}$ from fits (I) and (III) are more consistent between themselves (and less consistent with zero) than the best-fit values of $c_{1}$ coming from fits (II) and (III). We would like to note that these findings are corroborated by analogous fits of the $a \mu_{q}$ dependence of the static potential at fixed values of $r / a$, i.e. in situations where no interpolation in $r / a$ is involved.

\footnotetext{
${ }^{7}$ We are indebted to R. Sommer for very useful discussions on this point.
} 
Table 14

Results for masses and decay constants in the charged pseudoscalar channel, PCAC quark mass and $Z_{\mathrm{V}}$

\begin{tabular}{llllll}
\hline Run & $a \mu_{q}$ & $a m_{\mathrm{PS}}$ & $a f_{\mathrm{PS}}$ & $a m_{\mathrm{PCAC}}$ & $Z_{\mathrm{V}}\left(\mu_{q}\right)$ \\
\hline$B_{1 a}$ & 0.0040 & $0.13587(68)$ & $0.06531(40)$ & $-0.00001(27)$ & $0.6114(85)$ \\
$B_{1}$ & 0.0040 & $0.13623(65)$ & $0.06459(37)$ & $+0.00017(17)$ & $0.6136(19)$ \\
$B_{2}$ & 0.0064 & $0.16937(36)$ & $0.07051(35)$ & $-0.00009(17)$ & $0.6096(21)$ \\
$B_{3}$ & 0.0085 & $0.19403(50)$ & $0.07420(24)$ & $-0.00037(20)$ & $0.6115(22)$ \\
$B_{4}$ & 0.0100 & $0.21004(52)$ & $0.07591(40)$ & $-0.00097(26)$ & $0.6209(25)$ \\
$B_{5}$ & 0.0150 & $0.25864(70)$ & $0.08307(34)$ & $-0.00145(42)$ & $0.6165(22)$ \\
\hline
\end{tabular}

The results for the first three quantities come from a fit to a $4 \times 4$ submatrix with operators $\bar{d} \gamma_{5} u$ and $i \bar{d} \gamma_{0} \gamma_{5} u$, while for $Z_{\mathrm{V}}$ we used the full $6 \times 6$ matrix. Note that the difference between the first two rows is just the length of the simulation. The time range of the fit was always $10-23$ and the $\chi^{2} /$ d.o.f. was always smaller than one.

We conclude that the mass dependence is well described by the ansatz (I) and remark that an almost identical central value for $r_{0} / a$ at the chiral point is obtained from the ansatz (III), which also allows for a linear term in $a \mu_{q}$. The ansatz (II) gives a central value for $r_{0} / a$ at the chiral point lying two standard deviations above that from the ansatz (I). Finally we note that, if the ansatz (I) for the $\mu_{q}$-dependence of $r_{0} / a$ is used, the relative statistical accuracy of our determination of $r_{0} / a$ in the chiral limit is better than $1 \%$.

\section{Some selected results}

In this section we present results for quantities related to the pseudoscalar (PS) channel. This includes, apart from charged and neutral PS masses and decay constants, also the renormalisation constant $Z_{\mathrm{V}}$, which is specifically relevant to maximally twisted mass QCD.

\subsection{Charged and neutral pseudoscalar masses}

\subsubsection{Charged pseudoscalar meson mass}

To extract the charged PS mass $m_{\mathrm{PS}}$ we consider the correlation functions discussed in Section 2.1. We refer to this section and Section 4.1 for a detailed discussion of how the correlation functions are evaluated and the errors are estimated. The results for the charged PS masses can be found in Table 14.

In order to make the effect of the longest runs at $\mu=0.004$ visible, we quote the results for run $B_{1 a}$ and the complete run $B_{1}$ separately. While for $B_{1 a}$ we have 1811 measurements made in the cyclic way explained in Section 4.2, there are 895 measurements for $B_{1}$ performed moving the source time-slice randomly through the lattice. Even though in the latter case we have fewer measurements, they are more decorrelated because the single measurements are more separated in Monte Carlo time and because the distance of the position of the sources in Euclidean time for two consecutive measurements is on average larger. It is reassuring to see that results and errors are consistent between the two sets of data within errors. From this comparison it is also clear that moving the source time-slice randomly through the lattice is the most convenient of the two methods.

In Fig. 5 we show examples for effective masses in the PS channel at our lightest quark mass, extracted from the PS correlation function (with insertion of the $\bar{d} \gamma_{5} u$ operator) only. We plot the data for the three different choices of the interpolating operators, namely local-local, local-fuzzed, and fuzzed-fuzzed. One can see in Fig. 5 that the three different operators give compatible results from $t / a \approx 10$ on. Hence we are confident that the ground state energy dominates for $t / a>9$ and we chose the fit range accordingly.

We also attempted to determine the energy of the first excited state of the PS meson from a 2-state fit to the $6 \times 6$ matrix of

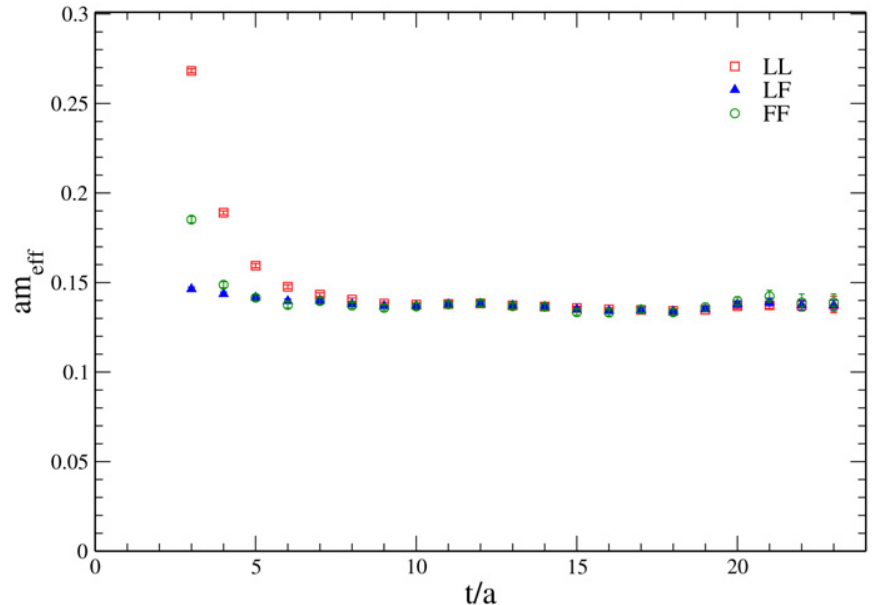

Fig. 5. Effective mass for the pseudoscalar channel from $B_{1}$ lattice data. The effective masses obtained using 3 different interpolating operators as described in the text are shown.

\section{Table 15}

Neutral pseudo scalar meson masses and decay constants at $\beta=3.9$ measured from every 10 trajectories at $\mu_{q}=0.004$ and every 20 at $\mu_{q}=0.0085$, as indicated; $a m_{\mathrm{PS}, \mathrm{conn}}^{0}$ is the mass extracted from the quark-connected correlators only

\begin{tabular}{llllll}
\hline Run & $a \mu_{q}$ & $N_{\text {meas }}$ & $a m_{\mathrm{PS}, \text { conn }}^{0}$ & $a m_{\mathrm{PS}}^{0}$ & $a f_{\mathrm{PS}}^{0} / Z_{\mathrm{A}}$ \\
\hline$B_{1}$ & 0.0040 & 888 & $0.212(3)$ & $0.109(7)$ & $0.089(3)$ \\
$B_{3}$ & 0.0085 & 249 & $0.259(3)$ & $0.169(11)$ & $0.106(4)$
\end{tabular}

correlators. Even though we were unable to determine the first excited level in a reliable way from an unconstrained fit, fixing it to the theoretical value ( 3 times the ground state mass), as expected in the continuum limit, does allow an acceptable fit.

This result is quite interesting, as with maximally twisted Wilson quarks one expects on general grounds also an $\mathrm{O}\left(a^{2}\right)$ contamination from the $\pi^{0}(\mathbf{0}) \pi^{ \pm}(\mathbf{0})$ two-pion state. Such a contamination becomes negligible, if compared to the expected three-pion state one in the continuum limit (taken at fixed quark mass). It should also be observed that when the pion mass is decreased, the twopion contribution remains negligible with respect to the three-pion one until $a^{2} \Lambda_{\mathrm{QCD}}^{2} \ll e^{-m_{\mathrm{PS}} t}$. For the range of $m_{\mathrm{PS}}$ and $t$ values relevant for our data we find that two-pion contamination effects can hardly be detected despite our (small) statistical errors (see Fig. 5).

\subsubsection{Neutral pseudoscalar meson mass}

As discussed in Section 3, the neutral PS meson can be created by interpolating fields that at maximal twist and in the twisted basis are of the form $\bar{\chi} \chi$ and $\bar{\chi} \gamma_{0} \gamma_{5} \chi$. We evaluate the correlator (both quark-connected and quark-disconnected pieces) with each of these operators at source and sink (also with local and fuzzed variants, thus giving a $4 \times 4$ matrix of correlators) as described above and in Appendix B. We fit this correlator matrix to one or more states in the usual way. Based on our study of autocorrelations (see Section 4), we compute statistical errors by a bootstrap analysis on blocked data where each block includes measurements taken on configurations corresponding to a segment of MC history 80 trajectories long.

Our results for the neutral PS meson are shown in Table 15. Compared to Ref. [1], we have increased statistics at $\mu_{q}=0.004$ and we have employed the more refined fitting procedure explained above. In particular we used $4 \times 4$ matrix of correlators rather than a $2 \times 2$ matrix. We also include results at a second $\mu_{q}$ value, $\mu_{q}=0.0085$. In order to show the contribution of the quark-disconnected component to the neutral PS meson mass determination, we show appropriate ratios in Fig. 6. 


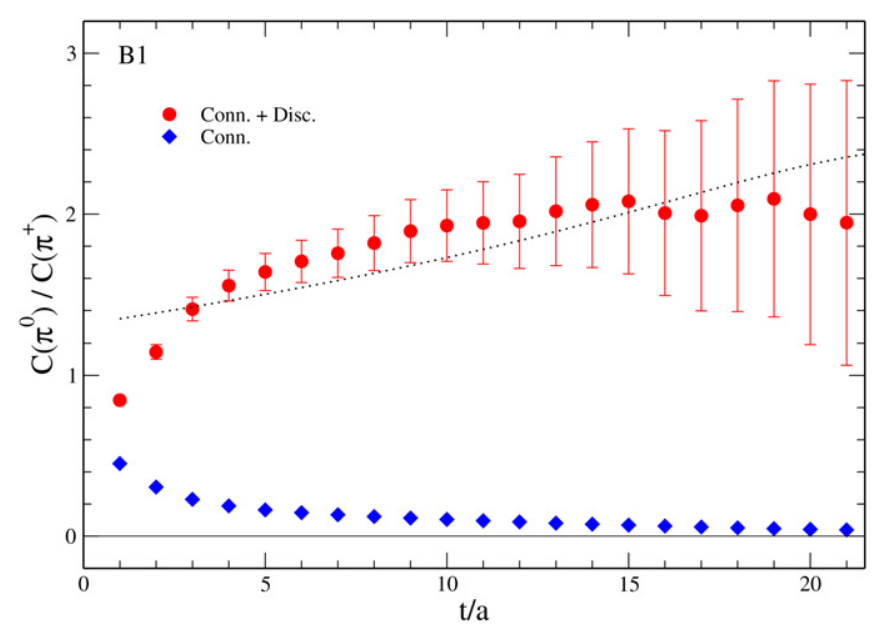

Fig. 6. Ratios of correlators of neutral to charged pseudoscalar (PS) mesons. The connected component of the neutral meson correlator is shown, as well as the total, which includes the disconnected contribution. The dotted curve illustrates the behaviour of the ratio of the correlator as obtained from our fitted values of the charged and neutral PS masses.

We have also evaluated the energies of neutral PS mesons with momentum $2 \pi / L$ (recall $L / a=24$ ), obtaining (by use of the continuum dispersion relation $\left.E^{2}=(2 \pi / L)^{2}+m^{2}\right)$ mass values consistent with those shown in Table 15.

The non-zero momentum results have the advantage that no vacuum subtraction is needed for the neutral PS meson correlator and this provides a crosscheck of the approach we employed. For example, at $\mu_{q}=0.004$ we obtain an energy of 0.309 (27) which correspond to a mass $0.164_{-60}^{+47}$ in lattice units.

\subsubsection{Pseudoscalar meson mass splitting and related topics}

Concerning the PS meson mass, it is well known that with maximally twisted Wilson fermions, even in the theory with $N_{f}=2$ degenerate quarks we consider here, there is difference of order $a^{2}$ (at fixed quark mass) between the neutral and the charged PS meson mass. Moreover the latter is very mildly affected by cutoff effects, once maximal twist is implemented in the optimal way of Section 1.1, as it follows from the formula $m_{\mathrm{PS}}^{2}-\left.m_{\mathrm{PS}}^{2}\right|_{\text {cont }}=$ $\mathrm{O}\left(a^{2} \mu_{q}\right)+\mathrm{O}\left(a^{4}\right)$ proved in Refs. [13,14,42]. Finally a lattice chiral perturbation theory analysis (see, e.g., Refs. $[13,43,44]$ ) shows that in the small $\mu_{q}$ region the difference between the squared neutral and charged PS masses tends to an $\mathrm{O}\left(a^{2}\right)$ quantity

$r_{0}^{2}\left(\left(m_{\mathrm{PS}}^{0}\right)^{2}-\left(m_{\mathrm{PS}}\right)^{2}\right) \simeq c\left(a / r_{0}\right)^{2}$,

which can be related to one coefficient (usually called $c_{2}$ ) of the chiral effective Lagrangian of (twisted and untwisted) Wilson fermion lattice QCD. From our results we estimate $c=-5.0(1.2)$ and $c=-6.7(2.8)$ respectively at $\mu_{q}=0.004$ and $\mu_{q}=0.0085$. These determinations are consistent within errors, as expected. Moreover, unlike in the case of lattice formulations with different gluonic actions [45], the sign of $c$ is now in agreement with the first order phase transition scenario $[44,46,47]$, as predicted by lattice chiral perturbation theory.

Some results for the coupling of the neutral PS meson field to the divergence of the neutral axial current are also given in Table 15 . The values of $f_{\mathrm{PS}}^{0}$ are found to be quite close to those obtained for the charged PS meson (see $f_{\mathrm{PS}}$ in Table 14), if the estimate $Z_{A}=0.76(2)[48,49]$ is employed. The differences between the central values are compatible with zero within 1 to 1.5 standard deviations of $f_{\mathrm{PS}}^{0}$ (which is the least precise result of the two).

This good consistency between the two channels is in striking contrast with the large cutoff effect present in $m_{\mathrm{PS}}^{0}$, which we observe as a large difference (about $50 \mathrm{MeV}$ at $a \mu_{q}=0.0040$ and

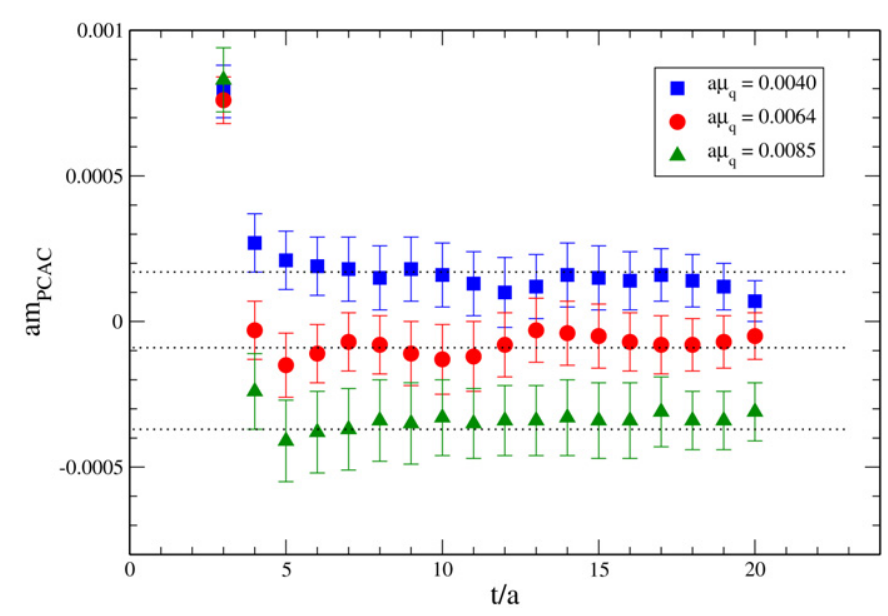

Fig. 7. PCAC quark masses versus time separation (from Eq. (3)) for three values of the mass parameter, $\mu_{q}=0.004, \mu_{q}=0.0064$ and $\mu_{q}=0.0085$. The lines represent the central values of the PCAC quark mass from a fit to a $4 \times 4$ matrix of observables for a $t$-range $10-23$.

$\left.a \mu_{q}=0.0085\right)$ between its value and that of its charged counterpart, $m_{\mathrm{PS}}$. Theoretical arguments providing an explanation for the reasons why a large lattice artifact affects only $m_{\mathrm{PS}}^{0}$ were presented in Ref. [42] and will be further discussed in a forthcoming publication [50].

\subsection{PCAC mass}

As discussed in Section 1.1, we attempt to tune the value of $m_{\text {PCAC }}$ (see Eq. (3)) to zero at our minimal $\mu_{q}$ value, namely at $a \mu_{q}=0.004$. A definition of $m_{\mathrm{PCAC}}$ equivalent to Eq. (3) for time separations so large that the lowest PS meson state dominates, is given by

$m_{\mathrm{PCAC}}=\frac{m_{\mathrm{PS}}}{2} \frac{\left\langle 0\left|A_{0}^{a}\right| P S\right\rangle}{\left\langle 0\left|P^{a}\right| P S\right\rangle}, \quad a=1,2$.

These two matrix elements can be directly determined from a fit to the $4 \times 4$ matrix involving the interpolating operators $\bar{d} \gamma_{5} u$ and $i \bar{d} \gamma_{0} \gamma_{5} u$ (or from the fit to the full $6 \times 6$ matrix, see Section 2 ). The results we obtain when a $4 \times 4$ matrix is used are summarised in Table 14 and shown as a horizontal line in Fig. 7. It is important to notice that the condition discussed around Eq. (10) is fulfilled for all our simulation points, and in particular for $a \mu_{q}=0.004$.

In Fig. 7, we also illustrate the time dependence of the local determination of the PCAC quark mass through Eq. (3). We see that the values of $m_{\text {PCAC }}$ determined using Eq. (3) in this way and Eq. (32) agree very well between themselves, in the $t$-region where the ground state pseudoscalar meson dominates, as expected.

Compared to Ref. [1] we now have a result for $a m_{\text {PCAC }}$ available for the large statistics run $B_{1}$. It is reassuring that there is full consistency between the 5000 trajectory run and the run extended up to 10000 trajectories. This makes us confident that our error estimate is realistic. Our results for $a m_{\text {PCAC }}$ as a function of the bare quark mass $a \mu_{q}$ are illustrated in Fig. 8, where results from the full ensemble, $B_{1}$, and those from the smaller ensemble, $B_{1 a}$, are separately shown.

\subsection{Pseudoscalar decay constant and $Z_{\mathrm{V}}$}

Using the exact lattice (twisted basis) PCVC relation ${ }^{8}$

$\left\langle\partial_{\mu}^{*} \tilde{V}_{\mu}^{a}(x) O(0)\right\rangle=-2 \mu_{q} \epsilon^{3 a b}\left\langle P^{b}(x) O(0)\right\rangle, \quad a=1,2$,

\footnotetext{
8 We recall that at maximal twist the twisted basis vector current $V_{\mu}^{a}$ corresponds to the axial current $\epsilon^{3 b a} A_{\mu}^{\prime b}(a, b=1,2)$ in the physical quark basis.
} 




Fig. 8. The PCAC quark mass $a m_{\mathrm{PCAC}}$ as a function of $a \mu_{q}$.

where $\partial_{\mu}^{*}$ is the lattice backward derivative, $O$ a local lattice operator and $\tilde{V}_{\mu}^{a}(x)$ the 1-point-split vector current

$$
\begin{aligned}
\tilde{V}_{\mu}^{a}(x)= & \frac{1}{4}\left[\bar{\chi}(x) \tau_{a} U_{\mu}(x)\left(\gamma_{\mu}-r\right) \chi(x+\hat{\mu})\right. \\
& \left.+\bar{\chi}(x+\hat{\mu}) \tau_{a} U_{\mu}^{\dagger}(x)\left(\gamma_{\mu}+r\right) \chi_{\mu}(x)\right],
\end{aligned}
$$

we can also compute (in the charged meson channel) the pseudoscalar meson decay constant with no need of any renormalisation constant (see $[2,4,51]$ ) from the formula

$f_{\mathrm{PS}}=\frac{2 \mu_{q}}{m_{\mathrm{PS}}^{2}}\left|\left\langle 0\left|P^{a}\right| P S\right\rangle\right|, \quad a=1,2$.

Based on the exact relation (33) and noting that

$\partial_{\mu}^{*} \tilde{V}_{\mu}^{a}(x)=Z_{\mathrm{V}} \tilde{\partial}_{\mu} V_{\mu}^{a}(x)+\mathrm{O}\left(a^{2}\right)$,

where $V_{\mu}^{a}$ is the naive current defined in Eq. (A.1) and $\widetilde{\partial}_{\mu}$ the symmetric lattice derivative, we can determine the (scale-independent) renormalisation constant $Z_{\mathrm{V}}$ through $\left(a, b=1,2\right.$ and $\left.x_{0} \neq 0\right)$

$Z_{\mathrm{V}}\left(a \mu_{q}\right)=\frac{-2 \mu_{q} \sum_{\mathbf{x}} \epsilon^{3 a b}\left\langle P^{b}(x) P^{b}(0)\right\rangle}{\sum_{\mathbf{x}} \widetilde{\partial}_{0}\left\langle V_{0}^{a}(x) P^{b}(0)\right\rangle}$ and
$Z_{\mathrm{V}}=\lim _{\mu_{q} \rightarrow 0} Z_{\mathrm{V}}\left(a \mu_{q}\right)$

with only $\mathrm{O}\left(a^{2}\right)$ cutoff effects. The results for $a f_{\mathrm{PS}}$ extracted using Eq. (35) are collected in Table 14 and will be discussed further in the next section.

In the same table we quote the results for the determination of $Z_{\mathrm{V}}$ that is based on fitting the large time behaviour of Eq. (37) where the pion state dominates

$$
\begin{aligned}
& \frac{-2 \mu_{q} \sum_{\mathbf{x}} \epsilon^{3 a b}\left\langle P^{b}(x) P^{b}(0)\right\rangle}{\sum_{\mathbf{x}} \widetilde{\partial}_{0}\left\langle V_{0}^{a}(x) P^{b}(0)\right\rangle} \\
& \stackrel{x_{0} \text { large }}{\longrightarrow} \frac{2 \mu_{q} \epsilon^{3 a b}\left\langle\Omega\left|P^{b}\right| \pi^{b}(\mathbf{0})\right\rangle}{m_{\mathrm{PS}}\left\langle\Omega\left|V^{a}\right| \pi^{b}(\mathbf{0})\right\rangle} .
\end{aligned}
$$

In practice the relevant matrix elements are extracted from a factorising fit (see Eq. (14)) to a $(4 \times 4)$ matrix of correlators, with entries given by the expectation values of $\sum_{\mathbf{x}} P^{1}(x) P^{1}(0)$ and $\sum_{\mathbf{x}} V_{0}^{2}(x) P^{1}(0)$ with local-local and local-fuzzed operators.

The $a \mu_{q}$ dependence of $Z_{V}$ is everywhere very weak (see Fig. 9), which makes the extrapolation to the chiral point easy and almost irrelevant, and leads to a rather precise result: $Z_{\mathrm{V}}=$ $0.615(5)$. The quoted error is a conservative estimate of the total uncertainty on $Z_{\mathrm{V}}$, inferred from the data in the last column of Table 14 and their statistical errors.

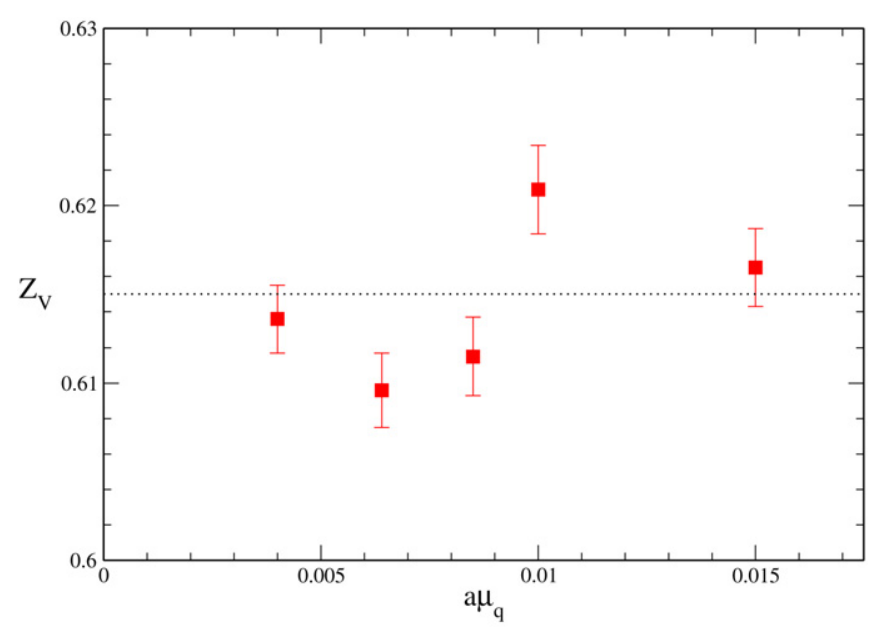

Fig. 9. Extrapolation of $Z_{V}$ to zero quark mass at $\beta=3.9$. The data are consistent within errors with the constant behaviour shown in the figure.

Another determination of $Z_{\mathrm{V}}\left(a \mu_{q}\right)$ is obtained by evaluating the ratios of correlators in the right-hand side of Eq. (37) for a number of time separations $\left(x_{0} / a \geqslant 10\right)$ and taking the average over them. This alternative method to evaluate $Z_{V}$ has been presented in Ref. [48]. Applying it, for instance, to the available ( $\sim 900)$ correlators computed on the whole ensemble $B_{1}$ yields the result $Z_{\mathrm{V}}\left(a \mu_{q}=0.0040\right)=0.6101(2)$. Both approaches, the one discussed in some detail above and the one of Ref. [48], provide precise determinations for $Z_{\mathrm{V}}$ and the virtues of both methods will be further discussed in Ref. [49].

\section{Chiral Perturbation Theory analysis of $\boldsymbol{f}_{\mathrm{PS}}$ and $\boldsymbol{m}_{\mathrm{PS}}$}

In this section we present the details of the comparison of our data with Chiral Perturbation Theory $(\chi \mathrm{PT})$ predictions. The main results have been already published in Ref. [1]. Here we provide further information about our fitting procedure and error analysis. The main goal of this section is to explain the fitting procedure and error determination using chiral perturbation theory. We will therefore only use the ensembles $B_{1}-B_{5}$, i.e. the ensembles that have already been discussed in Ref. [1]. For this limited set of data at only one value of the lattice spacing of $a=0.087 \mathrm{fm}$, we are not sensitive to higher order effects of chiral perturbation theory. We will therefore restrict ourselves to a 1-loop analysis of the data on $M_{\mathrm{PS}}$ and $f_{\mathrm{PS}}$. Nevertheless, we use the 2-loop chiral perturbation theory expressions and vary parameters of the corresponding formulae to see the possible effects, if the 2-loop order would be important to describe our data. In particular, this 2-loop investigation confirms that the here chosen dataset is indeed not sensitive to higher loop corrections.

Some preliminary results at larger volumes $(L / a=32)$ and finer lattice spacing $(\beta=4.05)$ have been already presented $[15,16]$. However, the present work is focused on the details of the analysis of the data points presented in Ref. [1]. The study of the volume and scaling dependence will be presented elsewhere. Notice, however, that, with respect to Ref. [1] we have a larger statistics at the smallest quark mass.

Our raw data for $a m_{\mathrm{PS}}$ and $a f_{\mathrm{PS}}$ are determined as described in Section 4.1. Results are reported in Table 14. As said above, there is no need to compute any renormalisation constant in order to make contact with the corresponding physical quantities.

In our $\chi$ PT based analysis we have to take into account finite size corrections because on our lattices at the lowest and next-tolowest $\mu_{q}$-values they turn out to affect $a m_{\mathrm{PS}}$ and, especially, $a f_{\mathrm{PS}}$ in a significant way. 
We have used continuum $\chi$ PT to describe consistently the dependence of the data both on the finite spatial size $(L)$ and on the bare quark mass $\left(\mu_{q}\right)$. This might look inappropriate in view of the existence of a large additive $\mathrm{O}\left(a^{2}\right)$ artifact in the neutral pion mass squared. ${ }^{9}$ However this is not so, because theoretical analyses carried out in the framework of lattice $\chi$ PT [13] and Symanzik expansion (complemented with soft pion theorems in the continuum theory) $[14,42]$ show that, if maximal twist is implemented as discussed in Section 7.2, the charged pion squared mass differs from its continuum counterpart only by $\mathrm{O}\left(a^{2} \mu\right)$ and $\mathrm{O}\left(a^{4}\right)$ terms, while the charged pion decay constant is affected by (chirally nonenhanced) discretisation errors of order $a^{2}$. Moreover the Symanzik expansion analysis is applicable for all spatial volumes $L^{3}$, provided $L$ is large enough to justify the use of soft pion theorems in the continuum theory at the quark mass of interest. This entails that also the $L$-dependence of the charged pion squared mass and decay constant is expected to be essentially continuum-like. These expectations are also supported by preliminary and still partial results we obtain at different lattice resolutions and different physical volumes $[15,16]$. Last but not least, the continuum $\chi$ PT formulae appear to describe well our data, as we are going to show below.

We fit the appropriate $\left(N_{f}=2\right)$ next-to-leading-order (NLO) $\chi$ PT formulae $[52,53]$

$m_{\mathrm{PS}}^{2}(L)=2 B_{0} \mu_{q}\left[1+\frac{1}{2} \xi \tilde{g}_{1}(\lambda)\right]^{2}\left[1+\xi \log \left(2 B_{0} \mu_{q} / \Lambda_{3}^{2}\right)\right]$,

$f_{\mathrm{PS}}(L)=F\left[1-2 \xi \tilde{g}_{1}(\lambda)\right]\left[1-2 \xi \log \left(2 B_{0} \mu_{q} / \Lambda_{4}^{2}\right)\right]$,

to our raw data for $m_{\mathrm{PS}}$ and $f_{\mathrm{PS}}$ simultaneously. Here ${ }^{10}$

$\xi=2 B_{0} \mu_{q} /(4 \pi F)^{2}, \quad \lambda=\sqrt{2 B_{0} \mu_{q} L^{2}}$.

The finite size correction function $\tilde{g}_{1}(\lambda)$ was first computed by Gasser and Leutwyler in Ref. [52] and is also discussed in Ref. [53] from which we take our notation (except that our normalisation of $f_{\pi}$ is $130.7 \mathrm{MeV}$ ). In Eqs. (39) and (40) next-to-next-to-leading order (NNLO) $\chi$ PT corrections are assumed to be negligible (this assumption is critically discussed in Section 7.1). The formulae above depend on four unknown parameters, $B_{0}, F, \Lambda_{3}$ and $\Lambda_{4}$, which will be determined by fitting to our data.

\subsection{Statistical errors}

In order to estimate the errors on the fit parameters it is important to account for both autocorrelation and cross-correlation of the data. We have exploited two different methods to do so.

\subsubsection{Method A}

The first method (see also [10]) consists in computing the full covariance matrix of our data for $a f_{\mathrm{PS}}$ and $\left(a m_{\mathrm{PS}}\right)^{2}$ and include it in the computation of $\chi^{2}$

$\chi^{2}=\sum_{i, j}\left(y_{i}-F_{i}\right) V_{i, j}^{-1}\left(y_{j}-F_{j}\right)$,

where $V$ is the covariance matrix ${ }^{11}$

$V_{i, j}=\operatorname{cov}\left(y_{i}, y_{j}\right)=\operatorname{cov}\left(\left(y_{i}-F_{i}\right)\left(y_{j}-F_{j}\right)\right)$,

\footnotetext{
9 Theoretical arguments have been presented [42] suggesting that this lattice artifact is an exceptional, though important, case, because it is related to the large value of a continuum matrix element appearing in the Symanzik expansion of the $\pi^{0}$-mass and does not stem from large coefficients multiplying the dimension five and six terms of the Symanzik effective Lagrangian.

10 We stress that $\xi$ defined here should not be confused with the continuum matrix element $\xi_{\pi}$ introduced in Eq. (5).

11 As we have data from independent simulations (ensembles $B_{1}$ to $B_{5}$ ), in the present case the covariance matrix will be block-diagonal with five blocks.
}

normalised so that the diagonal elements coincide with the squared standard error, and we have expressed the $\chi$ PT ansatz, Eqs. (39) and (40), in the form

$y_{i}=F_{i}(\vec{x}, \vec{\theta})$.

Here we denote by $y_{i}$ the primary measured quantities (in this section: $\left.y_{1}=\left(\left.a m_{\mathrm{PS}}\right|_{B_{1}}\right)^{2}, y_{2}=\left.a f_{\mathrm{PS}}\right|_{B_{1}}, y_{3}=\left(\left.a m_{\mathrm{PS}}\right|_{B_{2}}\right)^{2}, \ldots\right)$, by $\vec{x}$ the independent (error-free) variables (in this section: only $x=$ $a \mu_{q}$ ) and by $\vec{\theta}$ the parameters to be determined by the fit (here: $\left.\theta_{1}=2 a B_{0}, \theta_{2}=a F, \theta_{3,4}=\log \left(a^{2} \Lambda_{3,4}^{2}\right)\right)$. The error on the parameters are thus given by

$\left(\Delta \theta_{\alpha}\right)^{2}=\left(\nabla_{\theta_{\alpha}} F^{T} V^{-1} \nabla_{\theta_{\alpha}} F\right)^{-1}$.

The autocorrelations of $\left(a m_{\mathrm{PS}}\right)^{2}$ and $a f_{\mathrm{PS}}$ have been estimated both by data-blocking and by means of the $\Gamma$-method, as discussed in Section 4.1. Both approaches indicate (see Table 5) that by combining data into blocks of 32 measurements each (this corresponds always to more than $60 \mathrm{MC}$ trajectories) the resulting blocked data are safely uncorrelated. These blocked data are thus used to evaluate the covariance matrix, the $\chi^{2}$ and the errors on the fit parameters as discussed above (Eqs. (42)-(45)). In this way the possible effect of cross-correlations among the observables is included in the covariance matrix and therefore properly accounted for in the fit procedure.

In some of the checks that we are going to present below it will not always be possible to reduce the $\chi$ PT formulae to the form of Eq. (44). This happens in particular in the following cases: when computing directly $a f_{\mathrm{PS}}$ as a function of $a m_{\mathrm{PS}}$, when including the effects due to a non-vanishing am $\mathrm{PCAC}$, when including higher orders in Finite Size Effects (FSE) calculations, as computed in [53], or when we will eventually study the scaling dependence for different lattice spacings $a$. In all these cases the $\chi$ PT formulae can be expressed in the more general form $G_{i}(\vec{y}, \vec{x}, \vec{\theta})=0$ and the errors are given by the formula [54]: $\left(\Delta \theta_{\alpha}\right)^{2}=\left(\nabla_{\theta_{\alpha}} G^{T}\left(\nabla_{y} G V \nabla_{y} G^{T}\right)^{-1} \nabla_{\theta_{\alpha}} G\right)^{-1}$. These errors are obtained as propagation from the known errors on $\vec{x}$, therefore they do not depend on how good the fit is. The quality of the fit is expressed as usual by the quantity $\chi^{2} /$ d.o.f.

To provide a further check of possible effects of cross-correlation, the fits were also performed by dividing the data set into two subgroups each of half the size. The data for $a m_{\mathrm{PS}}$ were taken from one subgroup of gauge configurations and those for $a f_{\mathrm{PS}}$ from the other, ensuring in this way absence of cross-correlation. Errors scale as $\sqrt{2}$, i.e. as expected from halving the statistic, which indicates a negligible effect of cross-correlations in the full data set. Stability was also checked against different choices of subgroups. This result is confirmed by the observation that if we suppress the off-diagonal terms in the covariance matrix, our error bars are affected only at the percent level.

\subsubsection{Method $B$}

Method A is standard, and of course unbiased if for all observables the data are distributed in a Gaussian way (which we checked explicitly to be the case to a good approximation) and if the functions $F$ (or $G$ ) have a sufficiently linear behaviour around the relevant values of their arguments. An even safer estimate of the final errors can be obtained with the bootstrap method $[38,55]$.

To apply the bootstrap analysis method to our data set we proceed as follows. In order to account for autocorrelations we first form bins of 32 gauge configurations for each value of $\mu_{q}$. Out of the blocked data we generate 1000 bootstrap samples. The size of each sample is chosen as large as the full (blocked) data set. From the 1000 bootstrap samples we obtained 1000 observations for $2 a B_{0}, a F, \log \left(a^{2} \Lambda_{3,4}^{2}\right)$, and $\bar{l}_{3,4} \equiv \log \left(\Lambda_{3,4}^{2} / m_{\pi}^{2}\right)$, respectively. Error estimates are then computed as prescribed by the bootstrap 
Table 16

Comparison of fit results from Methods A and B

\begin{tabular}{lcc}
\hline & Method A & Method B \\
\hline $2 a B_{0}$ & $5.04(7)$ & $5.04(7)$ \\
$a F$ & $0.0522(7)$ & $0.0522(7)$ \\
$\log \left(a^{2} \Lambda_{3}^{2}\right)$ & $-1.90(11)$ & $-1.91(10)$ \\
$\log \left(a^{2} \Lambda_{4}^{2}\right)$ & $-1.00(4)$ & $-1.00(4)$ \\
$\chi^{2} /$ d.o.f. & $1.0 / 4$ & $0.9 / 4$ \\
$Q$ & 0.91 & 0.92 \\
\hline
\end{tabular}

method, i.e. by the standard deviation over the (equally weighted) 1000 samples. Incidentally we remark that this procedure takes the cross correlation between $a m_{\mathrm{PS}}^{2}$ and $a f_{\mathrm{PS}}$ correctly into account. In the 1000 fits we performed we have hence always used only the diagonal elements of the covariance matrix (fixed to their central values, i.e. to the square of the statistical errors on $a m_{\mathrm{PS}}^{2}$ and $a f_{\mathrm{PS}}$, see Table 14) as weights to evaluate the $\chi^{2}$ formula (42). In this specific application of the bootstrap method the error bars on the basic quantities $a m_{\mathrm{PS}}^{2}$ and $a f_{\mathrm{PS}}$ are still needed, since the observables of interest, the low energy constants (LEC), are defined through minimisation of the $\chi^{2}$ of the simultaneous fit to the $\chi$ PT formulae (39)-(40). Note that to safely employ the bootstrap method data need not have a Gaussian distribution, nor do the constraints, defined by the $\chi$ PT formulae, need to be linear. The bootstrap method may become expensive if single fits are significantly computer time demanding.

Both Methods A and B give consistent results, as shown in Table 16. In this paper we use the same setup as in Ref. [1], but we employ a somewhat larger statistics. The results are consistent. In addition to the error estimates we quote the value of $\chi^{2}$ and the merit figure of the fit defined via

$Q=1-P\left(\chi^{2} / 2\right.$, d.o.f. $\left./ 2\right)$,

where $P$ is the incomplete Gamma function [56].

\subsection{Discussion of systematic errors}

The error bars quoted in Table 16 are only statistical. As we also stressed in Ref. [1], a number of systematic effects are expected. Here we present some checks we performed in order to estimate the actual magnitude of these systematic effects.

As a first, simple check on the possible impact of neglected NNLO terms on the results presented in Table 16, we have also included the heaviest point (the one at $a \mu_{q}=0.0150$ ) in the standard fit to the formulae (39)-(40). In this case the results are still compatible with those in Table 16 within 1.7 standard deviations, but the $\chi^{2} /$ d.o.f. of the fit jumps from 0.24 to 1.7 . This increase of $\chi^{2} /$ d.o.f. is mainly due to the point at $a f_{\text {PS }}$ at $a \mu_{q}=0.0150$, as we noted already in Ref. [1]. The results of the fit are displayed in the second column of Table 17. This suggests that only the first four quark mass points should be used when comparing our data for $a f_{\mathrm{PS}}$ and $a m_{\mathrm{PS}}$ with NLO $\chi \mathrm{PT}$, as was done in Ref. [1].

It is also very interesting to see how much the tiny deviations from maximal twist corresponding to the (statistically compatible with zero) measured central values of $m_{\text {PCAC }}$ affect our results for the low energy constants discussed in this section. To address this question we introduce the definition of bare quark mass, $m_{q}=\sqrt{\left(Z_{A} m_{\mathrm{PCAC}}\right)^{2}+\mu_{q}^{2}}$, which holds for generic twist angle up to neglected $\mathrm{O}\left(a m_{\mathrm{PCAC}}\right)$ and $\mathrm{O}\left(a^{2}\right)$ terms. Moreover, in order to take into account the axial- $\tau_{3}$ transformation properties of the current entering the formal definition of $f_{\mathrm{PS}}$, at the same level of accuracy, the value of $a f_{\mathrm{PS}}$ should be corrected into $a f_{\mathrm{PS}} m_{q} / \mu_{q}$. We remark that this is obtained automatically if $f_{\mathrm{PS}}$ is evaluated from Eq. (35) with $\mu_{q}$ replaced by $m_{q}$-this can be related to the invariance of the operator $P^{1,2}$, a matrix element of which appears on
Table 17

Comparison of fit results from different setups, as explained in the text

\begin{tabular}{lcl}
\hline & Including $a \mu_{q}=0.015$ & $m_{q}=\sqrt{\left(Z_{\mathrm{A}} m_{\mathrm{PCAC}}\right)^{2}+\mu_{q}^{2}}$ \\
\hline $2 a B_{0}$ & $5.06(5)$ & $5.05(6)$ \\
$a F$ & $0.0508(5)$ & $0.0521(7)$ \\
$\log \left(a^{2} \Lambda_{3}^{2}\right)$ & $-1.93(6)$ & $-1.87(11)$ \\
$\log \left(a^{2} \Lambda_{4}^{2}\right)$ & $-0.89(2)$ & $-0.99(4)$ \\
$\chi^{2} /$ d.o.f. & $10.3 / 6$ & $0.55 / 4$ \\
$Q$ & 0.11 & 0.97 \\
\hline
\end{tabular}

\section{Table 18}

Percent Finite Size deviation $\left(m_{\mathrm{PS}}(L)-m_{\mathrm{PS}}(\infty)\right) / m_{\mathrm{PS}}(\infty)$ predicted by $\chi \mathrm{PT}$ for our data points. Note that nlo and nnlo include only the last order and not the previous one(s). According to Ref. [57], comparing nlo and nnlo (not lo and nlo) gives a reliable indication about the convergence of the expansion

\begin{tabular}{lllll}
\hline$a \mu_{q}$ & lo [52] & lo [53] & nlo [53] & nnlo [53] \\
\hline 0.0040 & $0.64 \%$ & $0.42 \%$ & $0.50 \%$ & $0.21 \%$ \\
0.0064 & $0.29 \%$ & $0.16 \%$ & $0.21 \%$ & $0.10 \%$ \\
0.0085 & $0.16 \%$ & $0.08 \%$ & $0.12 \%$ & $0.06 \%$ \\
0.0100 & $0.11 \%$ & $0.05 \%$ & $0.08 \%$ & $0.04 \%$ \\
\hline
\end{tabular}

Table 19

Same as in Table 18 , but for $\left(f_{\mathrm{PS}}(L)-f_{\mathrm{PS}}(\infty)\right) / f_{\mathrm{PS}}(\infty)$

\begin{tabular}{llll}
\hline$a \mu_{q}$ & lo [52] & lo [53] & nlo [53] \\
\hline 0.0040 & $-2.57 \%$ & $-1.68 \%$ & $-0.76 \%$ \\
0.0064 & $-1.15 \%$ & $-0.63 \%$ & $-0.30 \%$ \\
0.0085 & $-0.64 \%$ & $-0.32 \%$ & $-0.16 \%$ \\
0.0100 & $-0.44 \%$ & $-0.21 \%$ & $-0.11 \%$ \\
\hline
\end{tabular}

the right-hand side of Eq. (35), under axial- $\tau_{3}$ rotations. The results of this analysis, where we set $Z_{A}=0.76(2)$, as found at $\beta=3.9$ in Ref. [48], are shown in the last column of Table 17. It is reassuring to see that, thanks to the good precision we could reach in setting $m_{\text {PCAC }}$ to zero, the low energy constants of interest here are left essentially unaffected by this kind of correction.

We now consider the finite size corrections. In Ref. [1] we estimated them with the help of the formulae of Ref. [52]. A nice feature of these formulae is that they introduce no new parameter. However, they are only the first term of an expansion. Hence, the question is: how large is the residual uncertainty in FSE due to this truncation? To go beyond the first term in the framework of Ref. [52] is difficult. For the pseudo scalar mass the FSE corrections at two loops in $\chi$ PT have been computed in Ref. [53]. However, one can do better using the kind of $\chi$ PT expansion suggested in Ref. [57], for which results are also given in Ref. [53].

With the help of the results from Ref. [53] we can assess the stability of the prediction both by comparing the two approaches and by studying the convergence of the expansion of Refs. [53,57]. One should also notice that higher orders do introduce new parameters. Since it is not realistic to fit them, we will instead look at the stability of the prediction while changing those parameters in a "reasonable" range. The "reasonable" range is suggested in Ref. [53] and is based on phenomenological grounds.

To avoid confusion, we remark that the results of Ref. [52] are given as an expansion in powers of $1 / F_{0}$, while Ref. [53] uses an expansion in $1 / F_{\pi}$. This is the only reason why the first term of Ref. [53] does not coincide with Ref. [52].

In Tables 18 and 19 we show the percent deviation obtained using the formulae from Refs. [52] and [53] at different orders. Note that the new low energy constants (LECs) that at higher orders of $\chi$ PT are relevant for FSE are fixed to their central values estimated in Ref. [53]. See the comment below about their impact. To distinguish the expansion of the FSE effects from the usual $\chi$ PT expansion we will use a lower case notation (lo, nlo, nnlo) to denote the former one. The two expansions are of course related, but 
since the FSE also depend on the lattice size $L$, there is no reason to truncate the chiral expansion for FSE at the same order as the usual $\chi$ PT expansion. Here, for instance, we will use the NLO $\chi$ PT formulae, but we will compare FSE at lo, nlo and nnlo.

The convergence of the FSE expansion is expected to be good for all our data points since the smallest value of $m_{\mathrm{PS}} L$ is larger than 3. We recall that, according to Ref. [57], the comparison of lo and nlo is not a good indicator of the convergence of the expansion. This should be rather checked by comparing nlo and nnlo. According to all our estimates only the FSE at the lightest point $\left(a \mu_{q}=0.004\right)$ are relevant, while those at larger quark masses are always smaller than statistical errors. For instance, the deviations in $m_{\mathrm{PS}}$ are barely larger than its statistical errors (which amount to about $0.5 \%$ ). In order to check the dependence of the predicted FSE corrections on the LECs entering only at nlo, we changed randomly the value of the latter within the "reasonable" range suggested in Ref. [53]. We saw that nlo and nnlo FSE corrections are affected only at the level of about $20 \%$ (lo corrections are obviously unaffected) by such changes.

Up to this point we have only considered the $\chi$ PT at NLO (however corrections as high as nnlo are included in FSE calculations) implicitly assuming that NNLO contributions are negligible. This is reasonable, since $\chi \mathrm{PT}$ formulae with only NLO corrections yield a very good fit of the data at the lightest four quark masses, in spite of the fact that the expansion parameter, $\xi=2 B_{0} \mu_{q} /(4 \pi F)^{2}$, is not always very small. It is thus important to assess how much NNLO terms would affect our results.

The NNLO corrections relevant for $m_{\mathrm{PS}}$ and $f_{\mathrm{PS}}$ have been calculated in Ref. [58]. Here we use an expression which is easier to compare with lattice data, namely the one of Refs. [59,60] which reads

$$
\begin{aligned}
& m_{\mathrm{PS}}^{2}=M^{2}\left\{1+\xi \log \frac{M^{2}}{\Lambda_{3}^{2}}+\frac{17}{2} \xi^{2}\left[\log \frac{M^{2}}{\Lambda_{M}^{2}}\right]^{2}+4 \xi^{2} k_{M}+\mathrm{O}\left(\xi^{3}\right)\right\}, \\
& f_{\mathrm{PS}}=F\left\{1-2 \xi \log \frac{M^{2}}{\Lambda_{4}^{2}}-5 \xi^{2}\left[\log \frac{M^{2}}{\Lambda_{F}^{2}}\right]^{2}+4 \xi^{2} k_{F}+\mathrm{O}\left(\xi^{3}\right)\right\},
\end{aligned}
$$

where $\xi=2 B_{0} \mu_{q} /(4 \pi F)^{2}$ as before, $M^{2}=2 B_{0} \mu_{q}$ and

$$
\begin{aligned}
& \log \frac{\Lambda_{M}^{2}}{M^{2}}=\frac{1}{51}\left(28 \log \frac{\Lambda_{1}^{2}}{M^{2}}+32 \log \frac{\Lambda_{2}^{2}}{M^{2}}-9 \log \frac{\Lambda_{3}^{2}}{M^{2}}+49\right), \\
& \log \frac{\Lambda_{F}^{2}}{M^{2}}=\frac{1}{30}\left(14 \log \frac{\Lambda_{1}^{2}}{M^{2}}+16 \log \frac{\Lambda_{2}^{2}}{M^{2}}+6 \log \frac{\Lambda_{3}^{2}}{M^{2}}-6 \log \frac{\Lambda_{4}^{2}}{M^{2}}+23\right) .
\end{aligned}
$$

It is not realistic to attempt a fit of all the coefficients involved in the full NNLO expressions at least with the limited set of data used here. Rather we fix the parameters $\Lambda_{1}, \Lambda_{2}, k_{F}$ and $k_{M}$ to the values suggested in Ref. [53]. Since no estimate for $k_{M, F}$ is available, we take $k_{M, F}=0$. Redoing the fit in these conditions we can check how much NNLO terms change the results of Table 16. The new fit results are shown in the second column of Table 20. In order to further estimate to which extent these numbers are sensitive to a change in the parameters which were held fixed, we decided to change them one by one within the range proposed in Refs. [53,57], and perform a new fit for each one of these values. As for $k_{M}$ and $k_{F}$, it is difficult to tell what is a reasonable range, since, as we said, no estimate is available for them. On general grounds the values of $k_{M, F}$ are expected to be of $\mathrm{O}(1)$ and somewhat arbitrarily we assume a variability range $k_{M, F}= \pm 1$. This choice is also justified by the fact that larger variations quickly lead to very bad $\chi^{2}$. The results of this elaborated procedure are shown in columns 3 to 6 of Table 20. Most effects are not significant if compared to statistical errors, as they are never larger than a few standard deviations. It should be noted, however, that $\Lambda_{3}$ appears to be rather sensitive to $k_{M}$ and similarly $\Lambda_{4}$ to $k_{F}$. These

\begin{tabular}{|c|c|c|c|c|c|}
\hline & NNLO as in [53] & $\delta \Lambda_{1}= \pm 33 \%$ & $\delta \Lambda_{2}= \pm 5 \%$ & $k_{M}= \pm 1$ & $k_{F}= \pm 1$ \\
\hline $2 a B_{0}$ & $4.80(6)$ & $\begin{array}{r}-0.66 \% \\
3.44 \%\end{array}$ & $\begin{array}{r}-0.20 \% \\
0.26 \%\end{array}$ & $\begin{array}{l}3.2 \% \\
-2.5 \%\end{array}$ & $\begin{array}{l}0.07 \% \\
-0.12 \%\end{array}$ \\
\hline$a F$ & $0.0536(6)$ & $\begin{array}{c}0.60 \% \\
-1.7 \%\end{array}$ & $\begin{array}{r}0.16 \% \\
-0.19 \%\end{array}$ & $\begin{array}{l}-0.19 \% \\
0.21 \%\end{array}$ & $\begin{array}{l}1.9 \% \\
-2.1 \%\end{array}$ \\
\hline $\log \left(a^{2} \Lambda_{3}^{2}\right)$ & $-2.13(12)$ & $\begin{array}{l}-9.6 \% \\
-5.9 \%\end{array}$ & $\begin{array}{r}-1.2 \% \\
0.87 \%\end{array}$ & $\begin{array}{l}-29 \% \\
26 \%\end{array}$ & $\begin{array}{l}-1.3 \% \\
1.5 \%\end{array}$ \\
\hline $\log \left(a^{2} \Lambda_{4}^{2}\right)$ & $-1.00(5)$ & $\begin{array}{l}-4.6 \% \\
-0.35 \%\end{array}$ & $\begin{array}{r}-0.50 \% \\
0.34 \%\end{array}$ & $\begin{array}{l}1.3 \% \\
-1.3 \%\end{array}$ & $\begin{array}{l}24 \% \\
-26 \%\end{array}$ \\
\hline$\chi^{2} /$ d.o.f. & 0.085 & $\begin{array}{l}1.7 \\
0.15\end{array}$ & $\begin{array}{l}1.1 \\
0.82\end{array}$ & $\begin{array}{l}0.48 \\
1.8\end{array}$ & $\begin{array}{l}1.4 \\
0.73\end{array}$ \\
\hline
\end{tabular}
LECs can deviate by about $25 \%$ when setting $k_{M, F}$ to +1 or to -1 .
Table 20

Fit results, including NNLO $\chi$ PT

The second column shows the results obtained with the choice of $\Lambda_{1,2}$ suggested in [53] and $k_{M, F}=0$. The other columns give the percent correction due to changing the corresponding parameter in the indicated range. For each line, the upper (lower) number corresponds to the higher (smaller) boundary value of the interval.

We mention that changes of the LECs similar to those reported in Table 20 are also obtained if the NNLO terms in Eq. (46) are replaced by simple polynomial terms, like $\rho_{M, F} \xi^{2}$ (with no logarithms), and the free parameters $\rho_{M, F}$ are set to their best fit values.

\subsection{Comments}

In summary the discussion developed in this section shows that at least the systematic errors coming from the unknown NNLO terms involving $k_{M, F}$ may be significantly larger than the statistical ones, mostly because the adopted range of values was, to some degree, arbitrarily chosen. However, as already said above, using only the datasets $B_{1}-B_{5}$ a reliable estimate of systematic uncertainties on $B, F, \Lambda_{3}$ and $\Lambda_{4}$ from the NNLO corrections is not possible. A better assessment about the magnitude of NNLO effects will be attempted elsewhere [61] using ETMC data at different lattice spacings.

Although FSE to our simulation data turn out to be less than a few percent, we have made a special effort to compute them quite accurately, because their impact on LECs cannot be neglected, as their magnitude is comparable to the size of our statistical errors. The computation of FSE made in Ref. [53] represents a considerable improvement on the classical estimate of Ref. [52], as uncertainties on the extra LECs entering the former computation at high orders have little impact on the results. Actually, the validity of the predictions of FSE from $\chi$ PT can be checked by performing simulations on lattices of increasing size in physical units. Preliminary results have been presented in Ref. [15].

\section{Summary}

In this paper we have illustrated and discussed a number of details concerning unquenched simulations of $N_{f}=2$ mass degenerate Wilson quarks at maximal twist. We have explained in Section 1 our criterion on how to tune the theory to maximal twist. In particular, we provided theoretical arguments for our choice of $m_{\mathrm{PCAC}} / \mu_{q} \leqslant 0.1$ and showed that an error $\Delta m_{\mathrm{PCAC}} / \mu_{q} \leqslant 0.1$ is appropriate for this purpose. Useful formulae for quark bilinears and their physical interpretation in different quark bases (twisted and physical) are collected in Appendix A.

We have then discussed in Section 2 the methods we have used to compute charged meson correlators emphasising the effectiveness of employing (fuzzed) stochastic time-slice sources in the so-called "one-end trick". We have demonstrated that this method complemented by a random choice of the source location leads to 
a significant noise reduction, at least for two-point correlators in the meson sector.

The computation of neutral mesons and, in particular, quarkdisconnected contributions has been described in Section 3 and in the corresponding Appendix B. We have spelled out the reasons for using stochastic volume sources which can be employed in combination with efficient variance reduction methods. All these technical improvements have allowed us to compute quark-disconnected contributions on our sets of unquenched gauge configurations to an acceptable accuracy.

In Section 4 we have illustrated the main features of the MC algorithms used in our simulations showing that the resulting autocorrelation times are small enough to allow for a trustworthy error analysis of physical observables. We also explain how our error analysis of the data was performed owing to the use of $\Gamma$ - and binning-methods.

The force parameter $r_{0}$ can serve as an important physical quantity to check the scaling behaviour towards the continuum limit. We have provided in Section 5 a comprehensive discussion of the methods we have used to extract $r_{0}$ on our configurations. It turns out that with the present data an accuracy of better than $1 \%$ can be reached for $r_{0}$ in the chiral limit. It is also found that $r_{0}$ has a mild quark mass dependence which is consistent with being quadratic in $\mu_{q}$.

Various results for the charged and neutral pseudoscalar masses, the untwisted PCAC quark mass and the renormalisation constant $Z_{\mathrm{V}}$ are collected in Section 6. In particular, we show "effective mass" plots demonstrating the stability of the Euclidean time plateaux, which enables us to extract precise results for mesonic quantities.

Finally, we have detailed in Section 7 how our $\chi$ PT analysis of the data on $m_{\mathrm{PS}}$ and $f_{\mathrm{PS}}$ has been carried out, explaining how we get errors on the fitted low energy constants of the effective chiral Lagrangian, $B_{0}, F, \Lambda_{3}$ and $\Lambda_{4}$. In addition, we have analysed the effects of higher orders in $\chi$ PT on the stability of fit parameters and discussed the finite size effects.

We consider the present paper as a technical reference work of our collaboration. The methods described here have been and will be extensively used in our ongoing future research on lattice QCD employing maximally twisted Wilson fermions.

\section{Acknowledgements}

We thank all other members of the ETMC for very valuable discussions and for a most enjoyable and fruitful collaboration. We also gratefully acknowledge discussions with D. Bećirević and $\mathrm{N}$. Christian. The computer time for this project was made available to us by the John von Neumann-Institute for Computing on the JUMP and Jubl systems in Jülich and apeNEXT system in Zeuthen, by UKQCD on the QCDOC machine at Edinburgh, by INFN and CNRS on the apeNEXT systems in Rome, by BSC on MareNostrum in Barcelona (www.bsc.es) and by the Leibniz Computer centre in Munich on the Altix system. We thank these computer centres and their staff for technical advice and help. On QCDOC we have made use of Chroma [62] and BAGEL [63] software and we thank members of UKQCD for assistance. For the analysis we used among others the R language for statistical computing [64].

This work has been supported in part by the DFG Sonderforschungsbereich/Transregio SFB/TR9-03, DFG project JA 674/5-1 and the EU Integrated Infrastructure Initiative Hadron Physics (I3HP) under contract RII3-CT-2004-506078. We also thank the DEISA Consortium (co-funded by the EU, FP6 project 508830), for support within the DEISA Extreme Computing Initiative (www.deisa.org). G.C.R. and R.F. thank MIUR (Italy) for partial financial support under the contracts PRIN04 and PRIN06. V.G. and D.P. thank MEC (Spain) for partial financial support under grant FPA2005-00711.

\section{Appendix A. Quark bilinear operators in the twisted basis}

We give in this appendix the expression of a number of bare quark bilinear operators that are relevant for the topics of this paper. The operators are expressed in terms of (i) simple composite fields (recall $\gamma_{5}=\gamma_{0} \gamma_{1} \gamma_{2} \gamma_{3}$ and $\sigma_{\mu \nu}=i / 2\left[\gamma_{\mu}, \gamma_{\nu}\right]$ ) in the twisted quark basis, where the fermionic action takes the form (1),

$S^{0}(x)=\bar{\chi}(x) \chi(x), \quad P^{\alpha}(x)=\bar{\chi}(x) \gamma_{5} \frac{\tau^{\alpha}}{2} \chi(x)$,

$A_{\mu}^{\alpha}(x)=\bar{\chi}(x) \gamma_{\mu} \gamma_{5} \frac{\tau^{\alpha}}{2} \chi(x), \quad V_{\mu}^{\alpha}(x)=\bar{\chi}(x) \gamma_{\mu} \frac{\tau^{\alpha}}{2} \chi(x)$,

$T_{\mu \nu}^{\alpha}(x)=\bar{\chi}(x) \sigma_{\mu \nu} \frac{\tau^{a}}{2} \chi(x), \quad T_{\mu \nu}^{0}(x)=\bar{\chi}(x) \sigma_{\mu \nu} \chi(x)$

and (ii) the twist angle $\omega$, where $\tan \omega=\mu_{q} /\left(m_{0}-m_{\text {crit }}\right)$ and $a m_{\text {crit }}$ is determined as discussed in Section 1.1. The expressions we get are

$A_{\mu}^{\prime \alpha}= \begin{cases}\cos (\omega) A_{\mu}^{\alpha}+\epsilon^{3 \alpha \beta} \sin (\omega) V_{\mu}^{\beta} & (\alpha=1,2), \\ A_{\mu}^{3} & (\alpha=3),\end{cases}$

$V_{\mu}^{\prime \alpha}= \begin{cases}\cos (\omega) V_{\mu}^{\alpha}+\epsilon^{3 \alpha \beta} \sin (\omega) A_{\mu}^{\beta} & (\alpha=1,2), \\ V_{\mu}^{3} & (\alpha=3),\end{cases}$

$P^{\prime \alpha}= \begin{cases}\cos (\omega) P^{3}+i \frac{1}{2} \sin (\omega) S^{0} & (\alpha=3), \\ P_{\alpha} & (\alpha=1,2),\end{cases}$

$S^{\prime 0}=\cos (\omega) S^{0}+2 i \sin (\omega) P^{3}$,

$T_{\mu \nu}^{\prime \alpha}= \begin{cases}T_{\mu \nu}^{\alpha} & (\alpha=1,2) \\ \cos (\omega) T_{\mu \nu}^{3}-i \frac{1}{2} \epsilon^{\mu \nu \lambda \rho} \sin (\omega) T_{\lambda \rho}^{0} & (\alpha=3) .\end{cases}$

These expressions follow from the relation between twisted basis $(\chi)$ and physical basis $(\psi)$ quark fields, which (see Eq. (1) and Ref. [3]) reads

$\chi=e^{-i \gamma_{5} \tau^{3} \omega / 2} \psi, \quad \bar{\chi}=\bar{\psi} e^{-i \gamma_{5} \tau^{3} \omega / 2}$,

and the (obvious) definitions of the bare primed operators in terms of physical basis quark fields $(\alpha=1,2,3)$

$A_{\mu}^{\prime \alpha}=\bar{\psi}(x) \gamma_{\mu} \gamma_{5} \frac{\tau^{\alpha}}{2} \psi(x), \quad V_{\mu}^{\prime \alpha}=\bar{\psi}(x) \gamma_{\mu} \frac{\tau^{\alpha}}{2} \psi(x)$,

$P^{\prime \alpha}=\bar{\psi}(x) \gamma_{5} \frac{\tau^{\alpha}}{2} \psi(x), \quad T_{\mu \nu}^{\prime \alpha}(x)=\bar{\psi}(x) \sigma_{\mu \mu} \frac{\tau^{a}}{2} \psi(x)$,

$S^{\prime 0}=\bar{\psi}(x) \psi(x)$.

All these bare operators renormalise multiplicatively, with the exceptions of $P^{\prime 3}$ and $S^{\prime}$, which undergo an additive mixing with the identity (cubically divergent for $P^{\prime 3}$, quadratically divergent and vanishing as $\mu_{q} \rightarrow 0$ for $S^{\prime 0}$ ). For the expression of renormalisation constants as functions of $\omega$ and the renormalisation constants of standard Wilson quark bilinears and further details, see Ref. [3]. It should be remarked that substantial simplifications occur for $\omega= \pm \pi / 2$ (maximal twist) in almost all formulae above. Moreover at maximal twist also the formulae for renormalisation constants [3] get much simpler than at generic $\omega$.

\section{Appendix B. Evaluation of disconnected loops}

The quark-disconnected (simply "disconnected" in the following for brevity) components of correlators are intrinsically noisier than the connected components, so it is essential to evaluate them as accurately as possible. For this purpose we need to compute the disconnected loops at every $t$ value and for as many gauge configurations as possible. This can be achieved by using the stochastic source methods as we now discuss. The goal of the approach is to have an error arising from the stochastic nature of the method 
which is smaller than the intrinsic variability associated with varying $t$ and gauge configuration. If this is achieved, then the stochastic error is negligible in the sense that any further improvement in the signal can only be obtained if more gauge configurations are employed.

As discussed in Section 2.1, the basic idea is to use stochastic sources $(\xi)$ having in general support on the whole lattice and solve the linear system for the quantities

$\phi=M^{-1} \xi$,

where $M$ is the lattice Dirac matrix for a given flavour. The equation above is the same as Eq. (16), with the omission of the noise sample label $r$ (to lighten notation). Note also that in this appendix the normalisation of $M$ is taken such that, if $D_{\text {latt }}$ denotes the twoflavour Dirac matrix in Eq. (1), then

$M_{u}=2 \kappa \operatorname{tr}\left[a D_{\text {latt }}\left(1+\tau_{3}\right) / 2\right]=A+H, \quad A=1+2 \kappa a \mu_{q} i \gamma_{5}$,

with $H$ the usual Wilson first-neighbour hopping matrix. It follows that

$\sum\left[\xi^{*} X \phi\right]_{R}=\sum X M^{-1}+$ noise

where the symbol $[\ldots]_{R}$ refers (as in Section 2.1) to the average over $R$ samples of the stochastic source, the symbol $\sum$ denotes the sum over colour, spin and space-time indices and $X$ can be (almost) any structure we wish to evaluate, like $\gamma$-matrix, gauge links, Fourier factor, $\cos (k x)$, etc. It should be observed that in evaluating the disconnected contributions to the neutral meson correlators each one of the two quark loops arising from Wick contractions must be averaged over completely independent samples of stochastic sources for the purpose of avoiding unwanted biases. Moreover, for each quark loop diagram, the sum in Eq. (B.3) is restricted to one single time-slice, while still ranging over all colour, spin and space indices.

A method we employed to reduce the variance of the stochastic noise without much additional computational effort is the hopping-parameter method [25]. This relies on the observations that the first four terms in the hopping parameter expansion of $\sum X M^{-1}$ can be easily evaluated exactly on each gauge configuration and that replacing their stochastic estimates with the exact values significantly reduces the variance. In fact, writing $M_{u}$ (see Eq. (B.2)) in the form $M_{u}=(1+H B) A$, where $B=1 / A$, one easily obtains the identity

$1 / M_{u}=B-B H B+B(H B)^{2}-B(H B)^{3}+\left(1 / M_{u}\right)(H B)^{4}$,

which can be used to give

$$
\begin{aligned}
\sum X / M_{u}= & \sum\left\{X \left(B-B H B+B(H B)^{2}-B(H B)^{3}\right.\right. \\
& \left.\left.+\left(1 / M_{u}\right)(H B)^{4}\right)\right\} .
\end{aligned}
$$

The last term in Eq. (B.5) can be evaluated stochastically because

$X\left(1 / M_{u}\right)(H B)^{4}=\lim _{R \rightarrow \infty}\left[\xi^{*}(H B)^{4} X \phi\right]_{R}$.

Since $H^{\dagger}=\gamma_{5} H \gamma_{5}$ and $\gamma_{5}$ commutes with $B$, the last formula can be rewritten in the form

$X\left(1 / M_{u}\right)(H B)^{4}=\lim _{R \rightarrow \infty}\left[\left(\gamma_{5}\left(B^{\dagger} H\right)^{4} \gamma_{5} \xi\right)^{*} X \phi\right]_{R}$.

Thus four extra multiplications of the source $\xi$ by $B^{\dagger} H$ are needed. This is a negligible overhead compared to the inversion needed to obtain $\phi$. The first four terms in Eq. (B.5) do not involve $1 / M_{u}$ and can be, as said above, evaluated straightforwardly for any choice of $X$. For a local operator $X$, the only non-zero contributions are from the first term if $X$ is proportional to 1 or $\gamma_{5}$ and the third term if $X$ is proportional to $\gamma_{5}$. For a non-local operator $X$ whose length of spatial path is more than four lattice hops (as used in this paper), the first four terms are all zero.

This variance reduction method reduces the standard error of the stochastic samples by a factor of 1.5 or more in our case. This is valuable (it saves a factor $2-3$ in computational time), but for twisted mass QCD a much more powerful method is also available, although it applies only to the case $\sum X\left(1 / M_{u}-1 / M_{d}\right)$. This last method can be, and has been indeed, used in many important applications, essentially all those where one has to evaluate correlators with insertions of neutral meson operators of the form (in the twisted basis) $\bar{\chi} \Gamma \tau_{3} \chi$, with any Dirac matrix $\Gamma$ and the flavour matrix $\tau_{3}$. Interpolating fields of this type occur, e.g., in the two-point correlators for $\eta^{\prime}, f_{0}$ and $\pi^{0}$ mesons (actually only one of the possible operators for $\pi^{0}$ ), as one can see from the table for the (twisted basis) neutral meson operators reported in Section 3.

The powerful method alluded above relies on combining the identities

$\left(M_{d}-M_{u}\right)=-4 i \kappa a \mu_{q} \gamma_{5}$

and

$\left(1 / M_{d}\right)\left(M_{d}-M_{u}\right)\left(1 / M_{u}\right)=1 / M_{u}-1 / M_{d}$

to get

$1 / M_{u}-1 / M_{d}=-4 i \kappa a \mu_{q}\left(1 / M_{d}\right) \gamma_{5}\left(1 / M_{u}\right)$.

The latter relation already serves as a method of variance reduction because the explicit (small, in our simulations) factor of $a \mu_{q}$ reduces the magnitude of the fluctuations. On top of that, an even more important point is that the right-hand side of Eq. (B.10) can be evaluated very effectively with the help of the "one-end-trick" $[19,20]$ and no further inversions. In fact, since $M_{u}^{\dagger}=\gamma_{5} M_{d} \gamma_{5}$, one has

$\sum X\left(1 / M_{u}-1 / M_{d}\right)=-4 i \kappa a \mu_{q} \sum X \gamma_{5}\left(1 / M_{u}\right)^{\dagger}\left(1 / M_{u}\right)$,

which can be evaluated with noise/signal ratio of $\mathrm{O}(1)$ via

$\sum X\left(1 / M_{u}-1 / M_{d}\right)=-4 i \kappa a \mu_{q} \sum\left[\phi^{*} X \gamma_{5} \phi\right]_{R}+$ noise,

where (we recall) $\phi=\left(1 / M_{u}\right) \xi$ and $\phi^{*}=\xi^{*}\left(1 / M_{u}\right)^{\dagger}$. Apart from the explicit sum denoted by $\sum$, the right-hand side of this formula contains an implicit sum over the space-time indices of the stochastic source $\xi$ in $\phi$ and $\phi^{*}$, which contributes to reduce the variance as it receives contributions from the whole lattice (spacetime) volume.

To give an idea of the effectiveness of the method based on Eq. (B.12) we consider, as an example, the special case $X=i \gamma_{5}$, where one obtains

$\sum i \gamma_{5}\left(1 / M_{u}-1 / M_{d}\right)=4 \kappa a \mu_{q} \sum\left[\phi^{*} \phi\right]_{R}+$ noise.

At $\beta=3.9$ and $\mu_{q}=0.004$ (ensemble $B_{1}$ ) the method based on Eq. (B.13) yields an error which turns out to be 6 times smaller than what would be obtained with a conventional stochastic volume source. From the measured stochastic contribution to the signal, as well as the observed total fluctuation, one can extract the intrinsic variation stemming from the statistical fluctuations of the gauge field. The goal of the stochastic method is to have errors arising from the stochastic method which are negligible compared to the intrinsic (gauge) noise. This we achieve, finding that the stochastic contribution to the total error has a standard deviation which is $2 / 3$ of the standard deviation arising from the intrinsic variation of the signal. In the example, above we employed 24 stochastic sources (with no components set to zero), resulting in a cost of 24 inversions, per gauge configuration. Note that a similar number of inversions is needed to compute the (quark-connected) charged meson correlators. 
We thus find that this variance reduction method, where applicable, is very powerful and effectively reduces the stochastic noise in the neutral meson correlators, making it smaller than the intrinsic noise coming from the fluctuations of the gauge field.

\section{Appendix C. $\Gamma$-method and data-blocking}

In this appendix, we discuss the $\Gamma$-method and the datablocking procedure we have used to estimate the statistical errors of our physical observables.

\section{C.1. $\Gamma$-method}

In this section, for completeness, we just recall the basis of the $\Gamma$-method introduced in [24]. In the case of a primary stochastic variable with "true value" $A$ (the symbol $A$ will also be used to denote the observable itself), a suitable estimator of the error on the ensemble average $\bar{a}$, i.e. its standard deviation $\sigma_{\bar{a}}$, is given by ${ }^{12}$

$\sigma_{\bar{a}}^{2}=\frac{1}{N} \sum_{n=-W}^{W} \Gamma_{\bar{a}}(n)$,

where $N$ is the number of measurements, $2 W+1 \ll N$ is the number of consecutive measurements used in the estimation (measurement "window") and

$\Gamma_{\bar{a}}(n)=\frac{1}{N-|n|} \sum_{i=1}^{N-|n|}\left(a^{i}-\bar{a}\right)\left(a^{i+|n|}-\bar{a}\right)$.

Here $\Gamma_{\bar{a}}(n)$ represents the straightforward estimator of the autocorrelation function $\Gamma_{A}(n)=\left\langle\left(a^{i}-A\right)\left(a^{i+|n|}-A\right)\right\rangle$ (the index $i$ in $a^{i}$ labels the individual measurements, while $\langle\ldots\rangle$ denotes the theoretical expectation value).

The integrated autocorrelation time is conventionally defined for primary quantities as in Eq. (25) and estimated by (see Eq. (C.1))

$\tau_{\text {int }}(\bar{a})=\frac{1}{2 \Gamma_{\bar{a}}(0)} \sum_{n=-W}^{W} \Gamma_{\bar{a}}(n) \equiv \frac{N \sigma_{\bar{a}}^{2}}{2 \bar{\sigma}_{a}^{2}}$,

Note that $\Gamma_{\bar{a}}(0) \equiv \bar{\sigma}_{a}^{2}$, see Eq. (C.2), is an estimate of the a priori variance of $A$.

The $\Gamma$-method can also be applied to the analysis of secondary observables, $F=f(A)$, where $f$ denotes a non-linear function of several primary observables, $A \equiv\left\{A_{1}, A_{2}, \ldots\right\}$. A typical example is the case where $A$ is given by the values of two-point hadron correlators at different time separations, with different smearing levels, etc., while $F$ is a suitable estimator of the hadron mass; of course, the details of the function $F=f(A)$ depend on the specific choice of the estimator, e.g., on the form of the fit ansatz for the correlators and the range of time separations employed in the fit. The main point here is that the deviation of any given finite-statistics estimate of $F, \bar{F} \equiv f(\bar{a})$, from the true value $f(A)$ can be approximated, in the limit of large statistics, by retaining the first term of the Taylor expansion of $f(\bar{a})$ around $f(A)$, i.e. by writing

$f(\bar{a})-f(A) \simeq \sum_{\alpha} \frac{\partial f(A)}{\partial A_{\alpha}}\left(\bar{a}_{\alpha}-A_{\alpha}\right)$,

where $\alpha$ is the index labelling the primary quantities, $A_{\alpha}$, upon which $f$ depends. This remark suggests to define a new quantity, $A_{f}$, which is a simple linear combination of primary quantities, and the corresponding finite-statistics estimate, $\bar{a}_{f}$, via the formula

$A_{f} \equiv \sum_{\alpha} \frac{\partial f(A)}{\partial A_{\alpha}} A_{\alpha}, \quad \bar{a}_{f} \equiv \sum_{\alpha} \frac{\partial f(A)}{\partial A_{\alpha}} \bar{a}_{\alpha}$,

\footnotetext{
12 For a discussion of these issues, see [65] and references therein.
}

where $\bar{a}_{\alpha}$ is the ensemble average of the primary stochastic variable $a_{\alpha}$ (with "true value" $A_{\alpha}$, as above). The variance of $\bar{F}=f(\bar{a})$ will be given by

$\sigma_{\bar{F}}^{2} \equiv\left\langle(f(\bar{a})-f(A))^{2}\right\rangle \simeq\left\langle\left(\bar{a}_{f}-A_{f}\right)^{2}\right\rangle$,

where the truncation of the Taylor series produces a relative bias $\mathrm{O}\left(N^{-1}\right)$ which can be neglected if the number of measurements $N$ is sufficiently large. A further bias of the same order of magnitude arises from the replacement $\left.\frac{\partial f(A)}{\partial A_{\alpha}} \rightarrow \frac{\partial f(A)}{\partial A_{\alpha}}\right|_{A=\bar{a}}$ in Eq. (C.5), which is done in practice to evaluate the first derivatives of $f$ with respect to the $A_{\alpha}$ 's. At this point $\sigma_{\bar{F}}^{2}$ is estimated by the formula that is obtained from Eq. (C.1) by replacing $\Gamma_{\bar{a}}(n)$ with

$\Gamma_{\bar{a}_{f}}(n)=\frac{1}{N-|n|} \sum_{i=1}^{N-|n|}\left(a_{f}^{i}-\bar{a}_{f}\right)\left(a_{f}^{i+|n|}-\bar{a}_{f}\right)$.

\section{C.2. Binning method}

In the case where a data-blocking (also called binning) procedure is instead adopted to account for autocorrelations, the binsize $B$ plays a role similar to that of the window $W$ in the $\Gamma$-method. The integrated autocorrelation time can thus be estimated, for sufficiently large values of $B$, by

$\tau_{\text {int }}(\bar{F}) \simeq \frac{\sigma_{\bar{F}}^{2}(B)}{2 \sigma_{\bar{F}}^{2}(1)}$,

where $\sigma_{\bar{F}}(B)$ denotes the jackknife estimate of the error on $\bar{F}$ (the mean value of $F$ ) that is obtained upon binning the measurements into blocks of size $B$.

\section{C.3. Error on the error: $\Gamma$-method vs data-blocking}

The estimator of Eq. (C.1) allows to reach the optimal compromise between the relative statistical error on $\bar{\sigma}_{\bar{a}}$ raising with $\sqrt{W}$, i.e. $\delta_{\text {stat }}\left(\bar{\sigma}_{\bar{a}}\right) / \bar{\sigma}_{\bar{a}} \sim \sqrt{W / N}$, and the relative systematic error (bias) decreasing exponentially with $W$, i.e. $\delta_{\text {syst }}\left(\bar{\sigma}_{\bar{a}}\right) \sim 1 / 2 \exp (-W / \tau)$, where $\tau$ is the characteristic time of slowest exponential mode of $\Gamma(n)$ (exponential autocorrelation time). An "optimal" value, $W_{\text {opt }}$, to be used as upper and lower bound for the sum in Eq. (C.1) can be obtained, e.g., by gradually increasing $W$ and inspecting "by eye" the onset of a plateau for $\bar{\sigma}_{\bar{a}}$ as a function of $W$, or requiring minimisation of the total error $\delta_{\text {tot }}=\delta_{\text {stat }}+\delta_{\text {syst }}$ [24]. Any valid criterion to truncate the sum necessarily corresponds to values of $W_{\text {opt }}$ for which the truncation errors become comparable with the statistical noise level on $\bar{\sigma}_{\bar{a}}$. This choice corresponds to an uncertainty on the error on $\bar{\sigma}_{\bar{a}}$ decreasing like $\sim \mathrm{O}\left(N^{-1 / 2}\right)$. For comparison we recall that the error on $\bar{\sigma}_{\bar{a}}$ upon use of the binning method would decrease only like $\sim \mathrm{O}\left(N^{-1 / 3}\right)$ [24]. In this case in fact the optimal choice corresponds to find a compromise between the relative statistical error on $\bar{\sigma}_{\bar{a}}$ (i.e. $\delta_{\text {stat }}\left(\bar{\sigma}_{\bar{a}}\right) / \bar{\sigma}_{\bar{a}} \sim \sqrt{B / 2 N}$ ) which increases with $\sqrt{B}$, and the relative systematic error (bias) (i.e. $\delta_{\text {syst }}\left(\bar{\sigma}_{\bar{a}}\right) \sim \tau / 2 B$ ) which decreases with $B^{-1}$.

\section{C.4. Further remarks}

In our error analysis carried out using the $\Gamma$-method, we decided to compare different criteria for the windowing procedure in order to test in this respect the robustness of our estimates. One method is given by the algorithm proposed in [24] which is close to optimal. A second criterion, which is slightly more conservative, consists in stopping the procedure as soon as $\bar{\Gamma}(n)$ becomes negative due to statistical fluctuations. In the 15 analysed cases (5 simulation points times 3 quantities), no systematic trend could be detected, with the two methods giving in most of the cases similar results. In the cases where we cyclically vary the time the wall 
source over the lattice (see Section 2.1) in order to restore translation invariance in the MC time, as required by the $\Gamma$-method, we average beforehand correlators over source cycles. ${ }^{13}$ We recall that the time-slice sequences used for the different ensembles and the value of $n=t_{p}$ are specified in Table 6 .

As already mentioned, the results of the $\Gamma$-method have been checked against binning procedures. For observables that are nonlinear functions of the primary quantities, the error estimates were obtained by combining the binning procedure with either bootstrap-sampling (with bin sizes $B=4,8,16,32$ in trajectory units) or standard jackknife. In the latter case the optimal bin size $B_{\text {opt }}$ was determined by requiring stabilisation of the estimate of the error (with $B_{\text {opt }} / \tau_{\text {int }} \approx 10$ or larger).

Different methods give in general comparable results. In the case of the binning+bootstrap procedure stabilisation of the error is however not always evident at the maximal bin size (32 in trajectory units). In particular the PCAC quark mass turns out to be affected by significant autocorrelations (see Section 4.2) and the binning procedure seems not to be able to give reliable estimates of the error. In this case indeed the results lie systematically below the estimates from the $\Gamma$-method. This can be understood recalling that the $\Gamma$-method leads to a more favourable dependence upon the number of measurements in the error attributed to the autocorrelation time than the binning method.

In view of these findings we have decided to use the $\Gamma$-method for the estimates of the errors on the plaquette and am $\mathrm{PCAC}$. Also the error estimates for fermionic quantities (other than $a m_{\mathrm{PCAC}}$ ) quoted in Section 6 come from this method. However, similar results are obtained if a binning based procedure is employed.

\section{Appendix D. Details of the static potential calculation}

In this appendix we provide some details on the way we compute the static quark-antiquark potential from our dynamical gauge configurations.

\section{D.1. Improved static action}

An improvement on the signal-to-noise ratio in the measurements of the Wilson loop can be obtained by employing suitably smeared temporal links. This can be viewed as a convenient modification (or improvement) of the action for static quarks [66,67] as long as gauge invariance, cubic and parity symmetries as well as the local conservation of the static quark number and the static quark spin symmetry are preserved. Under these conditions it is still guaranteed that the static quark action is free from $\mathrm{O}(a)$ cutoff effects [68]. The statistical improvement alluded above comes from a reduction of the noise-to-signal ratio essentially stemming from the fact that the modified static quark action obtained via the use of smeared temporal links induces a self-energy mass term with a significantly reduced coefficient in front of the $a^{-1}$ term [67]. For our measurements we use the so-called HYP-improved static quark action, which is obtained by replacing the temporal links $U_{4}\left(\vec{x}, x_{0}\right)$ in the Wilson loop by HYP-smeared links [69]

$U_{4}\left(\vec{x}, x_{0}\right) \rightarrow V_{4}^{\mathrm{HYP}}\left(\vec{x}, x_{0}\right)$.

The HYP-smearing requires the specification of three parameters $\vec{\alpha}=\left(\alpha_{1}, \alpha_{2}, \alpha_{3}\right)$ and, following Ref. [67], we choose $\vec{\alpha}=$ $(1.0,1.0,0.5)$ throughout our calculation.

13 We generically find correlations between consecutive measurements taken on well separated time-slices (e.g., by $\Delta t=12 a$ ) to be negligible.

\section{D.2. Spatial smearing}

The smoothing of the spatial links has the effect of reducing excited-state contamination in the correlation functions of the Wilson loops in the potential measurements. The operators which we measured in the simulations are constructed using the spatial APE smearing of Ref. [70]. The smoothing procedure we use consists in replacing every spatial link $U_{j}(x), j=1,2,3$, by itself plus a sum of its neighbouring spatial staples and then projecting back to the nearest element in the $\mathrm{SU}(3)$ group, i.e. we write

$$
\begin{aligned}
\mathcal{S}_{1} U_{j}(x) \equiv & \mathcal{P}_{\mathrm{SU}(3)}\left\{U_{j}(x)+\lambda_{s} \sum_{k \neq j}\left(U_{k}(x) U_{j}(x+\hat{k}) U_{k}^{\dagger}(x+\hat{j})\right.\right. \\
& \left.\left.+U_{k}^{\dagger}(x-\hat{k}) U_{j}(x-\hat{k}) U_{k}(x-\hat{k}+\hat{j})\right)\right\} .
\end{aligned}
$$

Here, $\mathcal{P}_{\mathrm{SU}(3)} Q$ denotes the unique projection onto the $\mathrm{SU}(3)$ group element $W$, which maximises $\operatorname{Re} \operatorname{Tr}\left(W Q^{\dagger}\right)$ for any $3 \times 3$ matrix $Q$. The smeared and $\mathrm{SU}(3)$ projected link $\mathcal{S}_{1} U_{j}(x)$ retains all the symmetry properties of the original link $U_{j}(x)$ under gauge transformations, charge conjugation, reflections and permutations of the coordinate axes. The whole set of spatially smeared links, $\left\{\mathcal{S}_{1} U_{j}(x), x \in L^{4}\right\}$, forms the spatially smeared gauge field configuration. An operator $\mathcal{O}$ which is measured on a $n$-times iteratively smeared gauge field configuration is called an operator at smearing level $\mathcal{S}_{n}$, indicated by the symbol $\mathcal{S}_{n} \mathcal{O}$. From our experience a good choice is to use $M=5$ different smearing levels $\mathcal{S}_{n}$, with $n=8,16,24,32,40$, and in all cases a smearing parameter $\lambda_{s}=0.25$.

\section{D.3. Static quark-antiquark pair correlators}

The matrix of static quark-antiquark pair correlation functions, each of which from a technical viewpoint corresponds to a spatially smeared and temporally improved Wilson loop, is constructed in the following way. At fixed $x_{0}$ we first form smeared string (i.e. quark-antiquark pair) operators along the three spatial axes, connecting $\vec{x}$ with $\vec{x}+r \hat{i}$, given by

$$
\begin{aligned}
\mathcal{S}_{n} V_{i}\left(\vec{x}, \vec{x}+r \hat{i} ; x_{0}\right)= & \mathcal{S}_{n} U_{i}\left(\vec{x}, x_{0}\right) \mathcal{S}_{n} U_{i}\left(\vec{x}+a \hat{i}, x_{0}\right) \ldots \\
& \mathcal{S}_{n} U_{i}\left(\vec{x}+(r-a) \hat{i}, x_{0}\right), \quad i=1,2,3,
\end{aligned}
$$

and improved temporal links at fixed $\vec{x}$, connecting $x_{0}$ with $x_{0}+t$, given by

$$
\begin{aligned}
V_{4}\left(x_{0}, x_{0}+t ; \vec{x}\right)= & V_{4}^{\mathrm{HYP}}\left(\vec{x}, x_{0}\right) V_{4}^{\mathrm{HYP}}\left(\vec{x}, x_{0}+a\right) \ldots \\
& V_{4}^{\mathrm{HYP}}\left(\vec{x}, x_{0}+(t-a)\right) .
\end{aligned}
$$

The smeared Wilson loop ${ }^{14}$ is then obtained by computing

$$
\begin{aligned}
\mathcal{W}_{l m}(r, t)= & \sum_{\vec{x}, x_{0}} \sum_{i=1}^{3} \operatorname{Tr} \mathcal{S}_{l} V_{i}\left(\vec{x}, \vec{x}+r \hat{i} ; x_{0}\right) V_{4}\left(x_{0}, x_{0}+t ; \vec{x}+r \hat{i}\right) \\
& \times \mathcal{S}_{m} V_{i}^{\dagger}\left(\vec{x}, \vec{x}+r \hat{i} ; x_{0}+t\right) V_{4}^{\dagger}\left(x_{0}, x_{0}+t ; \vec{x}\right) .
\end{aligned}
$$

Finally we define the matrix of static quark-antiquark pair correlators according to the formula

$C_{l m}(r, t)=\left\langle\mathcal{W}_{l m}(r, t)\right\rangle=C_{m l}(r, t)$,

where the average is over the configurations of the ensemble. Since we have chosen to employ $M=5$ different string operators (as

\footnotetext{
14 Let us remark that we measure the on-axis potential only, i.e. the potential extracted from Wilson loops having spatial extent in the direction of the lattice axes $\hat{i}, i=1,2,3$ only.
} 
discussed above) and we are concerned with correlators where two such operators are inserted, we end up with a $5 \times 5$ matrix of static quark-antiquark pair correlators.

\section{References}

[1] ETMC, P. Boucaud, et al., Phys. Lett. B 650 (2007) 304, hep-lat/0701012.

[2] ALPHA, R. Frezzotti, P.A. Grassi, S. Sint, P. Weisz, JHEP 0108 (2001) 058, heplat/0101001.

[3] R. Frezzotti, G.C. Rossi, JHEP 0408 (2004) 007, hep-lat/0306014.

[4] $\chi_{\mathrm{E}} \mathrm{F}, \mathrm{K}$. Jansen, A. Shindler, C. Urbach, I. Wetzorke, Phys. Lett. B 586 (2004) 432, hep-lat/0312013.

[5] $\chi_{\mathrm{L}} \mathrm{F}, \mathrm{K}$. Jansen, M. Papinutto, A. Shindler, C. Urbach, I. Wetzorke, Phys. Lett B 619 (2005) 184, hep-lat/0503031.

[6] $\chi_{\mathrm{L}}$, K. Jansen, M. Papinutto, A. Shindler, C. Urbach, I. Wetzorke, JHEP 0509 (2005) 071, hep-lat/0507010.

[7] F. Farchioni, et al., Eur. Phys. J. C 39 (2005) 421, hep-lat/0406039.

[8] F. Farchioni, et al., Eur. Phys. J. C 42 (2005) 73, hep-lat/0410031.

[9] F. Farchioni, et al., Phys. Lett. B 624 (2005) 324, hep-lat/0506025.

[10] F. Farchioni, et al., Eur. Phys. J. C 47 (2006) 453, hep-lat/0512017.

[11] P. Weisz, Nucl. Phys. B 212 (1983) 1.

[12] S. Aoki, O. Bär, Phys. Rev. D 70 (2004) 116011, hep-lat/0409006.

[13] S.R. Sharpe, J.M.S. Wu, Phys. Rev. D 71 (2005) 074501, hep-lat/0411021.

[14] R. Frezzotti, G. Martinelli, M. Papinutto, G.C. Rossi, JHEP 0604 (2006) 038, heplat/0503034.

[15] ETMC, C. Urbach, arXiv: 0710.1517 [hep-lat]

[16] ETMC, P. Dimopoulos, R. Frezzotti, G. Herdoiza, C. Urbach, U. Wenger, PoS LAT2007 (2007) 102, arXiv: 0710.2498 [hep-lat].

[17] C. Michael, A. McKerrell, Phys. Rev. D 51 (1995) 3745, hep-lat/9412087.

[18] J. Foley, K. Jimmy Juge, A. O’Cais, M. Peardon, S.M. Ryan, J.I. Skullerud, Comput Phys. Comm. 172 (2005) 145, hep-lat/0505023.

[19] UKQCD, M. Foster, C. Michael, Phys. Rev. D 59 (1999) 074503, hep-lat/9810021.

[20] UKQCD, C. McNeile, C. Michael, Phys. Rev. D 73 (2006) 074506, hep-lat/ 0603007.

[21] MILC, C. Aubin, et al., Phys. Rev. D 70 (2004) 114501, hep-lat/0407028.

[22] TXL, S. Gusken, et al., Phys. Rev. D 59 (1999) 114502.

[23] UKQCD, P. Lacock, A. McKerrell, C. Michael, I.M. Stopher, P.W. Stephenson, Phys. Rev. D 51 (1995) 6403, hep-lat/9412079.

[24] ALPHA, U. Wolff, Comput. Phys. Comm. 156 (2004) 143, hep-lat/0306017.

[25] UKQCD, C. McNeile, C. Michael, Phys. Rev. D 63 (2001) 114503, hep-lat/0010019.

[26] UKQCD, C. McNeile, C. Michael, Phys. Lett. B 556 (2003) 177, hep-lat/0212020.

[27] K. Jansen, hep-lat/0609012.

[28] S. Duane, A.D. Kennedy, B.J. Pendleton, D. Roweth, Phys. Lett. B 195 (1987) 216

[29] M. Hasenbusch, Phys. Lett. B 519 (2001) 177, hep-lat/0107019.

[30] M. Hasenbusch, K. Jansen, Nucl. Phys. B 659 (2003) 299, hep-lat/0211042.

[31] C. Urbach, K. Jansen, A. Shindler, U. Wenger, Comput. Phys. Comm. 174 (2006) 87, hep-lat/0506011.

[32] K. Jansen, A. Shindler, C. Urbach, U. Wenger, PoS LAT2005 (2006) 118, heplat $/ 0510064$

[33] J.C. Sexton, D.H. Weingarten, Nucl. Phys. B 380 (1992) 665.
[34] T. Takaishi, P. de Forcrand, Phys. Rev. E 73 (2006) 036706, hep-lat/0505020.

[35] R.C. Brower, T. Ivanenko, A.R. Levi, K.N. Orginos, Nucl. Phys. B 484 (1997) 353, hep-lat/9509012.

[36] T. Chiarappa, et al., hep-lat/0609023.

[37] ALPHA, R. Frezzotti, M. Hasenbusch, U. Wolff, J. Heitger, K. Jansen, Comput. Phys. Comm. 136 (2001) 1, hep-lat/0009027.

[38] B. Efron, R.J. Tibshirani, An Introduction to the Bootstrap, Monographs on Statistics and Applied Probability, vol. 57, CRC Press, 1993.

[39] R. Sommer, Nucl. Phys. B 411 (1994) 839, hep-lat/9310022.

[40] A. Hasenfratz, R. Hoffmann, F. Knechtli, Nucl. Phys. (Proc. Suppl.) 106 (2002) 418, hep-lat/0110168.

[41] F. Niedermayer, P. Rufenacht, U. Wenger, Nucl. Phys. B 597 (2001) 413, heplat/0007007.

[42] ETMC, R. Frezzotti, G. Rossi, arXiv: 0710.2492 [hep-lat].

[43] L. Scorzato, Eur. Phys. J. C 37 (2004) 445, hep-lat/0407023.

[44] G. Münster, JHEP 0409 (2004) 035, hep-lat/0407006.

[45] F. Farchioni, et al., PoS LAT2005 (2006) 033, hep-lat/0509036.

[46] S.R. Sharpe, J. Singleton, L. Robert, Phys. Rev. D 58 (1998) 074501, hep-lat/ 9804028.

[47] S.R. Sharpe, J.M.S. Wu, Phys. Rev. D 70 (2004) 094029, hep-lat/0407025.

[48] ETMC, P. Dimopoulos, et al., arXiv: 0710.0975 [hep-lat].

[49] ETMC, P. Boucaud, et al., 2008, in preparation.

[50] ETMC, P. Dimopoulos, et al., 2008, in preparation.

[51] R. Frezzotti, S. Sint, Nucl. Phys. (Proc. Suppl.) 106 (2002) 814, hep-lat/0110140.

[52] J. Gasser, H. Leutwyler, Phys. Lett. B 184 (1987) 83.

[53] G. Colangelo, S. Dürr, C. Haefeli, Nucl. Phys. B 721 (2005) 136, hep-lat/0503014.

[54] H. Britt, R. Luecke, Technometrics 15 (2) (1973) 233-247.

[55] A. Davison, D. Hinkley, Cambridge Series on Statistical and Probabilistic Mathematics, Cambridge University Press, 1997.

[56] W.V.W.H. Press, S.A. Teukolsky, B. Flannery, Numerical Recipes, Fortran, second ed., Cambridge University Press, 1992.

[57] G. Colangelo, S. Durr, Eur. Phys. J. C 33 (2004) 543, hep-lat/0311023.

[58] J. Bijnens, G. Colangelo, G. Ecker, J. Gasser, M.E. Sainio, Nucl. Phys. B 508 (1997) 263, hep-ph/9707291.

[59] H. Leutwyler, Nucl. Phys. Proc. Suppl. 94 (2001) 108, hep-ph/0011049.

[60] G. Colangelo, J. Gasser, H. Leutwyler, Nucl. Phys. B 603 (2001) 125, hep-ph/ 0103088.

[61] ETMC, P. Boucaud, et al., 2008, in preparation.

[62] R.G. Edwards, B. Joo, Nucl. Phys. Proc. Suppl. 140 (2005) 832, hep-lat/0409003.

[63] P. Boyle, http://www.ph.ed.ac.uk/ paboyle/bagel/Bagel.html.

[64] R Development Core Team, R: A language and environment for statistical computing, R. Foundation for Statistical Computing Vienna, Austria, ISBN 3-90005107-0, 2005.

[65] A.D. Sokal, Given at the Troisieme Cycle de la Physique en Suisse Romande, Lausanne, Switzerland, June 15-29, 1989.

[66] ALPHA, M. Della Morte, et al., Phys. Lett. B 581 (2004) 93, hep-lat/0307021.

[67] M. Della Morte, A. Shindler, R. Sommer, JHEP 0508 (2005) 051, hep-lat/ 0506008 .

[68] ALPHA, M. Kurth, R. Sommer, Nucl. Phys. B 597 (2001) 488, hep-lat/0007002

[69] A. Hasenfratz, F. Knechtli, Phys. Rev. D 64 (2001) 034504, hep-lat/0103029.

[70] APE, M. Albanese, et al., Phys. Lett. B 192 (1987) 163. 